\title{
Propriétés et territoires autochtones en Nouvelle-France
}

I - Contrôle territorial et reconnaissance de territoires

nationaux

\author{
Aboriginal territories and property in New France \\ I - The origins of land control and the recognition of national \\ territories
}

\author{
Propiedades y territorios indígenas en Nouvelle-France \\ I - Origen del control territorial y reconocimiento de \\ territorios nacionales
}

\section{Michel Morin}

Volume 43, numéro 2-3, 2013

URI : https://id.erudit.org/iderudit/1026107ar

DOI : https://doi.org/10.7202/1026107ar

Aller au sommaire du numéro

\section{Éditeur(s)}

Recherches amérindiennes au Québec

\section{ISSN}

0318-4137 (imprimé)

1923-5151 (numérique)

Découvrir la revue

\section{Citer cet article}

Morin, M. (2013). Propriétés et territoires autochtones en Nouvelle-France : i Contrôle territorial et reconnaissance de territoires nationaux. Recherches amérindiennes au Québec, 43(2-3), 59-75. https://doi.org/10.7202/1026107ar

\section{Résumé de l'article}

Cet article est publié en deux parties. La première partie, qui apparaît dans le présent numéro, porte sur les débats concernant l'origine du contrôle territorial et sur la reconnaissance des territoires nationaux. La seconde partie, qui sera publiée dans notre prochain numéro, traitera de la gestion des districts de chasse.

Dans le nord-est de l'Amérique du Nord, l'origine précolombienne des territoires familiaux autochtones a suscité une controverse chez les anthropologues, tout comme la possibilité que les Algonquiens aient élaboré par eux-mêmes des mesures de conservation des ressources fauniques. Pourtant, au début du XVII ${ }^{\mathrm{e}}$ siècle, les Français n'ont aucune difficulté à reconnaître l'existence de territoires dont l'accès est contrôlé par des nations autochtones et qui constituent la propriété collective de l'une d'entre elles; par conséquent, ses chefs en supervisent l'usage par ses membres. Avec le temps, les représentants du roi s'emploient à convaincre les peuples « frères » de s'accorder un droit mutuel de chasser sur leurs terres. Comme en Acadie, il existe en Nouvelle- France des " districts » de chasse bien délimités exploités sous la direction d'un chef de bande familiale. Les membres d'une autre bande ou les étrangers doivent obtenir la permission d'y chasser, quoique les incursions occasionnelles soient acceptées. À compter de 1660, des mesures de conservation sont observées dans la région des Grands Lacs et même dans celle $\mathrm{du}$ lac Champlain, mais au XVIII ${ }^{\mathrm{e}}$ siècle, elles ne semblent pas connues sur la Côte-Nord; cependant il paraît peu probable que les autochtones n'aient pas eu les connaissances suffisantes pour en élaborer eux-mêmes. Dans l'ensemble, leur con cep tion du territoire semble d'origine autochtone plutôt que française. Elle repose sur l'existence de limites nationales et de districts bien définis, même si les observateurs français ne cherchent pas à décrire ceux-ci précisément.
Tous droits réservés @ Recherches amérindiennes au Québec, 2013

Ce document est protégé par la loi sur le droit d'auteur. L'utilisation des services d'Érudit (y compris la reproduction) est assujettie à sa politique d'utilisation que vous pouvez consulter en ligne.

https://apropos.erudit.org/fr/usagers/politique-dutilisation/ 
Propriétés et territoires autochtones

en Nouvelle-France

I - Contrôle territorial et reconnaissance

de territoires nationaux*

\section{Michel Morin}

Faculté de droit, Université de Montréal

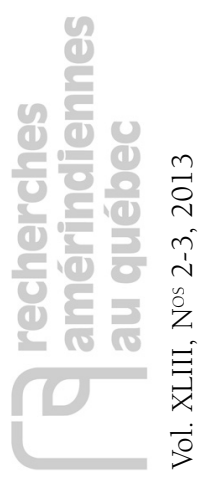

Ces sauvages sçavent les routes des bois et les connoissent comme nous savons les ruës d'une ville

(Raudot 1904 : 97)

L STATUT DES TERRITOIRES autochtones en Nouvelle-France a fait l'objet de nombreuses analyses divergentes, car la colonisation française repose sur deux principes potentiellement contradictoires. D'une part, l'affirmation théorique de la souveraineté française ne comporte pas l'intention d'éteindre les droits des peuples autochtones (Slattery 1979; Grammond 2003; Huppé 2009; Morin 2010). En effet, l'assujettissement de ces nations exige une modification formelle de leur statut, ainsi que l'adoption graduelle des règles du système juridique colonial (Morin 2013). Dans l'intervalle, ces peuples demeurent indépendants; c'est pourquoi des traités de paix ou des alliances militaires et commerciales peuvent être conclus avec eux.

D'autre part, contrairement à ce qui s'est produit dans les colonies britanniques, les représentants du roi de France ont la faculté de concéder à leurs compatriotes des terres situées sur le territoire traditionnel d'une nation autochtone. Sans être obligatoire

* La deuxième partie de cet article paraîtra dans notre prochain numéro. avant l'établissement des Français dans une région, une négociation ou une concertation demeurent indiquées, car le Roi réitère constamment sa volonté de maintenir des relations pacifiques avec ses alliés (ibid.). En outre, certains textes juridiques visent à assurer et à protéger les droits d'usage des autochtones. Dans cette perspective, ceux-ci ne peuvent être éliminés sans une décision expresse des autorités coloniales, telle la concession d'un titre foncier. Par contraste, certains auteurs considèrent que le pouvoir de concéder unilatéralement des terres dans l'immense territoire de la Nouvelle-France est difficilement compatible avec la reconnaissance de droits territoriaux autochtones (cf. Brun 1974; R. c. Côté, [1996] 3 R.C.S. 139, paragr. 42-54', Dawson 2005; Lavoie 2010a; Cavanagh 2014).

Il est bien connu que les autochtones ne connaissent pas la propriété individuelle de la terre et ne concevaient pas que celle-ci puisse être aliénée; tout au plus, l'occupation et l'exploitation du territoire pouvaient être partagées (voir Mailhot et Vincent 1982; Lajoie et al. 1996; Grammond 2003; Lacasse 2004; Bissonnette 2007). Néanmoins, le fait qu'historiquement ils aient collectivement exercé un contrôle sur leurs terres peut éclairer les débats actuels 
concernant la reconnaissance d'un droit ancestral ou issu de traité protégé par la constitution (voir l'article 35(1) de la Loi constitutionnelle de 1982; R. c. Marshall; R. c. Bernard, [2005]). De même, s'il existait des territoires familiaux en Nouvelle-France, ceux-ci pourraient éventuellement bénéficier d'une protection constitutionnelle (Behn c. Moulton Contracting Ltd. [2013]). Il importe donc de comprendre la nature des droits exercés par les autochtones sur leurs territoires à cette époque, sans procéder pour autant à une analyse détaillée de la situation de chaque peuple; en effet, il est souvent difficile d'établir un lien entre les noms employés au XVII ${ }^{\mathrm{e}}$ siècle et celui des peuples autochtones ou des lacs et rivières du Québec actuels (Dawson 2003, 2005; Chamberland et al. 2004). En outre, nous nous concentrerons sur les principaux peuples algonquiens qui, au début $\mathrm{du} \mathrm{XVII}^{\mathrm{e}}$ siècle, vivaient sur le territoire actuel du Québec, de l'Ontario et des provinces maritimes (principalement les Micmacs, les Innus, les Algonquins et les Cris, que nous désignerons aussi par les noms utilisés dans les sources citées).

Notre réflexion découle d'une analyse des comptes rendus des missionnaires et des récits d'explorateurs, qui constituent des sources aussi riches que volumineuses. Si ces documents peuvent traduire le biais d'observateurs européens, ils font souvent référence à des concepts n'ayant aucun équivalent dans la culture occidentale. Le fait que des auteurs différents décrivent ceux-ci de manière analogue nous semble augmenter grandement les chances que leurs descriptions soient fiables, ce qui n'exclut évidemment pas la possibilité qu'ils aient occasionnellement déformé ou méconnu la réalité. Ainsi, cherchent-ils rarement à comprendre précisément comment les territoires autochtones sont délimités ou comment des droits d'usage particuliers sont attribués. Néanmoins, ils font régulièrement référence au pouvoir de gestion exercé par les chefs autochtones sur les terres ancestrales de leur nation. Ces observations sont précieuses, car elles montrent que les Français sont bien conscients de l'occupation territoriale autochtone. De manière générale, elles semblent compatibles avec les données de la littérature anthropologique ou ethno-historique concernant les valeurs et les conceptions des autochtones, même si des écarts peuvent exister.

On sait que, dans le deuxième tiers du $\mathrm{xx}^{\mathrm{e}}$ siècle, des anthropologues et des économistes ont soutenu que l'idée d'un territoire exclusif autochtone ou familial est apparue à la suite des échanges avec les Européens dans le but de prévenir l'extermination des castors, le principal animal à fourrure faisant l'objet de ce commerce; d'autres anthropologues y voyaient plutôt des concepts d'origine précolombienne. Un retour sur cette controverse nous permettra de mettre en évidence l'ethnocentrisme qui colore en partie ces analyses, ainsi que les pièges qui guettent ceux ou celles qui analysent les renseignements disponibles pour cette période. Dans un deuxième temps, il nous faudra déterminer, à partir des explications fournies par les sources françaises, la nature du contrôle territorial exercé par les nations autochtones, ainsi que leur décision de mettre en commun les ressources fauniques à la suite de traités de paix. Nous examinerons ensuite la description des « districts» de chasse chez les Algonquiens du nordest de l'Amérique du Nord, les conflits observés après la raréfaction des ressources au tournant du $\mathrm{XVIII}^{\mathrm{e}}$ siècle, ainsi que les renseignements concernant les mesures de conservation observées à cette époque.

Notre objectif n'est pas tant de reconstituer la pensée et les valeurs des autochtones, mais plutôt de montrer qu'ils ont pu se faire comprendre des Français. À leur tour, ceux-ci ont été amenés à utiliser des concepts juridiques pour décrire l'occupation territoriale qu'ils observaient. Cela nous semble susceptible d'étayer les revendications contemporaines des peuples autochtones, même si cet aspect de la question ne sera pas discuté dans ce texte.

\section{LES DÉBATS CONCERNANT L'ORIGINE DU CONTRÔLE TERRITORIAL}

Dans la première moitié du $\mathrm{xx}^{\mathrm{e}}$ siècle, l'anthropologue Frank G. Speck a documenté l'existence de territoires de piégeage attribués à une famille et transmissibles de manière héréditaire. À son avis, cette forme de propriété existait avant le contact avec les Européens et avait survécu à la colonisation. Cette affirmation a été fortement contestée, en raison de l'absence de corroboration dans la documentation historique. Pour Eleanor B. Leacock, les zones de prélèvement des castors ne pouvaient être apparues avant le développement des échanges commerciaux avec les Européens. Cette explication est longtemps demeurée canonique; elle a notamment permis de justifier l'apparition de la propriété familiale chez les autochtones et, dans une certaine mesure, l'adoption par ceux-ci de mesures de préservation.

\section{LA CONTROVERSE ENTOURANT L'ORIGINE DES TERRITOIRES FAMILIAUX}

La discussion concernant l'apparition de la propriété a une origine ancienne qu'il convient de rappeler. En effet, la réflexion des juristes occidentaux a été grandement influencée par un texte de l'auteur romain Justin. Pour celui-ci, durant l'âge d'or de l'humanité, les êtres humains peuvent exploiter les ressources de la terre à leur guise, car il n'existe aucun pays et aucune forme de propriété; simplement, les fruits et le gibier appartiennent au premier qui s'en empare. Dès le début du XVII ${ }^{\mathrm{e}}$ siècle, Grotius s'inspire de cette idée. Il explique que l'apparition de limites tribales puis nationales est due à celle de l'élevage et de l'agriculture; par la suite, certaines sociétés reconnaissent la propriété individuelle ou familiale des terres cultivées. Même si telle semble être son opinion, il n'est pas absolument clair que, dans son esprit, un peuple puisse 
posséder collectivement des territoires de chasse, même s'il admet que les pasteurs possèdent leurs pâturages (Morin 1997 : 44-48).

En 1672, Pufendorf prend plus clairement position en faveur des autochtones. Il soutient qu'il existe un régime de communauté positive en vertu duquel les membres d'un peuple ou d'un groupe se réservent la jouissance d'un territoire donné, à l'exclusion des étrangers. Cette forme de propriété peut inclure les territoires de chasse. Elle s'oppose à la communauté négative, c'est-à-dire l'absence de toute appropriation ou le libre accès, comme dans le cas de l'air ou de la mer (ibid. : 52-53). Cette distinction demeure pertinente de nos jours. Dans la mesure où un peuple autochtone est doté de la personnalité juridique, il peut posséder collectivement des droits ancestraux ou un titre aborigène dont il déterminera les modalités d'exercice pour ses membres, par l'entremise de ceux qui ont le pouvoir de gérer ces biens collectifs (par exemple, les leaders des groupes de chasse) [Anker 2014; Leclair et Morin 2011, paragr. 64; Manitoba Metis Federation Inc. c. Canada 2013; Morin 1995; Vivier 2003: 236; Di Robilant 2012]. Il est également possible que certains individus détiennent une partie des droits d'une communauté ou soient autorisés à les exercer (voir Behn c. Moulton Contracting Ltd. [2013]). En France, sous l'ancien régime, il existe des propriétés collectives (boisés, pâturages etc.) appartenant à certaines communautés depuis des temps immémoriaux (voir Vivier 2003; Di Robilant 2012). Ce concept juridique nous semble compatible avec les connaissances actuelles sur les modes d'exploitation des territoires par les chasseurs-cueilleurs (Barnard et Woodburn 1998 : 4-15; Houde 2011).

À l'inverse de Pufendorf, en 1690 John Locke affirme que les autochtones d'Amérique n'établissent pas de limites entre les terres où ils chassent et pêchent (Locke 2002: 13-14). Dans son esprit, le droit d'exclure les autres d'un terrain apparaît uniquement après la création de gouvernements et de la monnaie (ibid. : 15-19). Cette innovation nécessite un accord au moins tacite par lequel certaines communautés ont renoncé au « droit naturel commun » d'utiliser les terres des autres nations. Dans les régions où il n'y a pas eu d'entente sur l'utilisation de l'argent, de nombreuses terres incultes demeurent soumises à ce droit d'usage universel, dans la mesure où il en existe suffisamment pour que tous subviennent à leurs besoins (ibid. : 21). En d'autres termes, les autochtones d'Amérique sont réputés connaître et accepter la règle permettant à toute personne de s'installer sur des terres qui ne sont pas cultivées ou sur lesquelles il n'y a pas d'habitation. Dans cette perspective, ils ne peuvent ni posséder ni revendiquer des terres ancestrales (voir Arneil 1996; Greer 2012; Yirush 2011; Tomlins 2010). Bien qu'elle ait été contestée par Wolff, cette thèse sera reprise par Vattel et finira par s'imposer au $\mathrm{XIX}^{\mathrm{e}}$ siècle parmi les internationalistes, avec l'apparition du concept juridique de terra nullius; auparavant, la question est demeurée très controversée (Morin 1997: 40-62 et 163-178; Morin 2010; Tomlins 2010 : 117-121).

Frank G. Speck s'est opposé à ce type de conception. En 1942, dans un texte coécrit avec Loren C. Eiseley, il présente l'aboutissement de recherches et de réflexions s'étendant sur près d'un demi-siècle, en tentant de répondre à certaines critiques formulées par des historiens (Speck et Eiseley 1942). À son avis, tous les peuples algonquiens vivant entre le cinquante-cinquième et le cinquante-deuxième degré de latitude, de l'Atlantique au lac Winnipeg, reconnaissent l'existence de territoires de chasse familiaux transmissibles dans la lignée paternelle, en particulier pour les animaux à fourrure, qui sont sédentaires. Plus au nord, en quittant les forêts touffues pour arriver dans la toundra, la chasse au caribou, qui mobilise un grand nombre d'individus, se pratique de manière communautaire sur l'ensemble du territoire, qui demeure donc collectif.

Les informateurs montagnais et naskapis de Speck décrivent les limites de leur territoire familial avec précision, avant même que la région ait été cartographiée par les autorités gouvernementales (ibid. : 215-218). Ils affirment qu'une intrusion sur leurs terres attirera le mauvais sort sur le fautif, mais ils n'envisagent pas de prendre euxmêmes des mesures de rétorsion (ibid. : 231). Il convient de souligner qu'à ce stade de sa réflexion, Speck ne confond aucunement la propriété individuelle de type occidental et les territoires de chasse familiaux. En outre, Speck et Eiseley concèdent qu'initialement, dans un territoire peu peuplé où la faune est abondante, un peuple autochtone n'a pas de raison de diviser le territoire en zones attribuées à des familles ou qui seront exploitées par différents chasseurs sur une base saisonnière. Lorsque les ressources se font plus rares, une telle attribution a lieu. La chasse communautaire de certaines espèces comme le caribou peut coexister avec une division en petits groupes familiaux pratiquant le piégeage, par exemple. Dès 1926, Speck affirme que cette évolution est inévitable, compte tenu des conditions environnementales, des pratiques de chasse et de la nécessité de permettre la reproduction des animaux (Speck 1926 : 327-328). Speck et Eiseley sont disposés à croire qu'à l'époque moderne, l'exploitation intensive des animaux à fourrure a stimulé l'apparition d'une "véritable propriété familiale », mais ils demeurent convaincus du fait que celle-ci est d'origine précolombienne (Speck et Eiseley 1942 : 238-240).

En 1937, Alfred G. Bailey soutient qu'au XvII ${ }^{\mathrm{e}}$ siècle, les échanges commerciaux avec les Français ont provoqué une intensification de la chasse au castor et une rareté de cette ressource. Cette activité requiert donc des expéditions qui se pratiquent en groupes plus petits qu'auparavant et dont la durée s'accroît significativement. Comme il est admis qu'un chasseur est propriétaire de l'animal qu'il 
abat, par extension on en vient à reconnaître la propriété des endroits où vivent les bêtes à fourrure; en outre, les Français contribuent à faire accepter l'idée d'un territoire familial, notamment ceux qui ont épousé des femmes autochtones (Bailey 1969 : 87-88). Bref, la transformation s'explique par l'arrivée des Européens et par l'adoption graduelle d'une mentalité individualiste par les autochtones (ibid. : xx-xxi).

En 1954, Eleanor B. Leacock soutient un point de vue semblable dans sa thèse de doctorat. Elle souligne qu'au dire de Speck, les territoires familiaux analysés n'ont pas un caractère permanent, ne sont pas aliénables et demeurent sujets au contrôle de la bande. Pour Leacock, il s'agit là d'une forme d'usufruit, plutôt que d'un droit de propriété. En outre, au début du XVII ${ }^{\mathrm{e}}$ siècle, le petit gibier a une importance mineure, car la survie du groupe dépend de la quantité d'orignaux ou de caribous abattus; il ne peut inciter les autochtones à se partager les zones de chasse. Surtout, la théorie de Speck suppose que ceux-ci ont ressenti le besoin d'adopter des mesures de conservation avant l'arrivée des Européens. Elle signale que, contrairement à ce qu'affirme Speck, les Naskapis partagent eux aussi leur territoire en zones familiales, sur une base saisonnière, même s'ils chassent en grands groupes dans la toundra (Leacock 1974 : 1-7).

Pour ce qui concerne le XVII ${ }^{\mathrm{e}}$ siècle, selon Leacock, les sources historiques ne révèlent rien qui s'apparente aux territoires familiaux observés par Speck trois siècles plus tard, même s'il existe certainement des territoires de bande, dont les limites sont toutefois mal définies et instables. En effet, les références aux limites des zones de chasse fréquentées par un groupe ne sauraient être assimilées à une forme de propriété. C'est seulement vers la fin $\mathrm{du} \mathrm{XVII}^{\mathrm{e}}$ siècle qu'on voit apparaître une référence très claire à la propriété des huttes de castor et à une allocation saisonnière des zones de chasse (ibid. : 15). Parallèlement, en hiver, la taille des bandes de chasseur diminue afin d'augmenter leur rayon d'action, ce qui facilite cette transformation (ibid. : 22). À cet égard, des recherches plus récentes ont établi que la chasse au petit gibier se pratiquait depuis longtemps en petits groupes, dont la taille n'a pas sensiblement diminué au cours de cette période (Morantz 1986: 65, 86).

En 1980, Leacock résume la position de ses adversaires de manière cinglante : ceux-ci «tentaient de réaliser par décret ce que les Jésuites avaient manqué au $\mathrm{XVII}^{\mathrm{e}}$ siècle : transformer les Montagnais, de peuple qui avait traditionnellement pratiqué le droit collectif à l'usage du territoire, en familles de propriétaires » (Leacock 1980:80). À son avis, « les relations de production chez les aborigènes du Canada subarctique étaient du type communisant » et le territoire y était considéré comme la "propriété collective de la population aborigène ». Il existait des bandes ou des groupes de chasseurs ayant chacun leur territoire et qui devaient demander une permission avant de pénétrer sur celui d'un autre (ibid. : 81). Toutefois, il était admis qu'en cas d'absolue nécessité, cela pouvait se faire immédiatement. Pour cette raison, les bandes, qui étaient composées de maisonnées, ne s'attribuaient « aucun droit exclusif sur le territoire » (ibid. : 83).

Leacock a été grandement influencée par la pensée marxiste, selon laquelle le communisme primitif constitue le stade premier de l'évolution sociale - une variante de l'idée immémoriale de l'âge d'or. En réalité, l'organisation sociale des Montagnais du début du XviI ${ }^{\mathrm{e}}$ siècle, avec ses expéditions guerrières et un réseau commercial étendu, n'est pas compatible avec une société élémentaire composée de simples bandes où les inégalités prononcées sont pratiquement absentes. Surtout, le processus de transformation et d'acculturation a été beaucoup plus long et complexe que ce que laisse entendre Leacock (Feit 2009: 57-60; Bender et Morris 1988: 7). Par ailleurs, des recherches récentes montrent bien que les leaders jouent depuis des temps très anciens un rôle important dans la répartition des groupes de chasse et dans le contrôle de l'utilisation de territoires, dont les limites sont relativement bien définies (voir Leroux 2009, 2010; Vincent 2010; Lacasse et Cabanes 2009). Il convient donc de se demander s'ils ont été appelés à mettre en place des mesures de conservation de la faune ou si cette idée leur a été inculquée par des Européens.

\section{LA CONTROVERSE ENTOURANT L'ORIGINE DES MESURES DE CONSERVATION DE LA FAUNE}

Lhypothèse selon laquelle les territoires familiaux seraient apparus chez les Montagnais à la suite de la rareté croissante des animaux à fourrure a rapidement suscité l'intérêt des économistes (Feit 2009: 61). En 1967, Harold Demsetz soutient que, si un territoire est exploité collectivement, aucun chasseur n'a intérêt à prendre des mesures de conservation, par exemple s'abstenir de tuer les femelles enceintes ou laisser certaines zones en jachère pendant une ou plusieurs années. En effet, un autre individu pourrait profiter de cette mesure, puisqu'il est libre de chasser partout dans le territoire de la bande. Toutefois, avec l'avènement de la traite des fourrures, celles-ci ont une valeur accrue aux yeux des autochtones, car elles permettent de se procurer des biens qu'ils ne peuvent produire, notamment les objets de métal. Il en résulte une exploitation intensive des ressources. À terme, celle-ci peut mener à l'extinction complète d'une ou plusieurs espèces, imposant ainsi un coût à tous les membres de la collectivité (Demsetz 1967 : 351-353). C'est ce qu'on appelle la «tragédie des biens communaux» (Hardin 1968).

Ce problème des externalités - le fait que le producteur ne supporte pas le coût des conséquences dommageables de son activité - expliquerait pourquoi, au cours du 
$\mathrm{XVII}^{\mathrm{e}}$ siècle, les autochtones ont créé des territoires familiaux qui les incitent à préserver les animaux à fourrure. Dans un régime de propriété collective, pour prendre de telles mesures, c'est-à-dire limiter les quantités chassées, l'accord de tous les membres est nécessaire, ce qui exige des négociations laborieuses et pose le problème de la surveillance des activités de chacun. Dans un régime de propriété familiale ou individuelle, ces difficultés sont bien moindres (Demsetz 1967 : 355-358)

En 2002, Demsetz souligne que les externalités ne sont pas le seul facteur expliquant l'apparition de la propriété individuelle. Au sein d'une communauté peu peuplée telle qu'un village, un système collectif de gestion peut être viable. Pour des chasseurs-cueilleurs, la propriété individuelle (ou familiale) n'offre pas forcément un grand intérêt. En effet, ils doivent parcourir de grandes distances, car une parcelle de terrain ne contient pas suffisamment de gibier ou de fruits pour les nourrir; en outre, il faut délaisser celles qui ont été exploitées récemment. Il n'y a aucun intérêt pratique à empêcher une intrusion temporaire d'étrangers. Surtout, la viande et les fruits se conservent et se transportent difficilement. En général, c'est plutôt l'avènement de l'agriculture qui fournit un incitatif à l'apparition de propriétés familiales (Demsetz 2002).

La typologie des économistes s'est également raffinée, notamment en tenant davantage compte du rôle positif que peut jouer la collectivité. Il faut de nos jours distinguer la propriété étatique, la propriété individuelle, la propriété collective d'un groupe particulier et l'absence de propriété ou l'accès libre (Cole et Ostrom 2012; Demsetz 2011; Krier 2009). Chacun de ces régimes peut s'avérer efficient dans des conditions données (Cole et Orstrom 2012 : 53-55; Flanagan et al. 2010 : 202-26). Toutefois, après avoir procédé à une revue des données historiques disponibles au XVII et au XVIII ${ }^{\mathrm{e}}$ siècle dans la vallée du Saint-Laurent et la région de la baie d'Hudson, Shepard Krech III montre que, pour les périodes plus anciennes, le souci de préserver les ressources fauniques semble avoir été largement absent lorsque celles-ci n'étaient pas menacées d'extinction, notamment en raison de la spiritualité autochtone. Cette prise de conscience aurait favorisé l'émergence des territoires familiaux (Krech III 1999 : 194-195). En outre, la mise en place de mesures de conservation serait due à l'influence des Européens (ibid. : 182-186).

Selon Harvey Feit, les stratégies de préservation pourraient tout aussi bien avoir été découvertes et diffusées par les autochtones, ce que leur mythologie paraît confirmer (2007: 64; voir aussi Carlos et Lewis 2006: 68; Savard 2004: 90). Par ailleurs, leur nécessité peut avoir été exagérée. Ainsi, dans une région où la population de castors est très dense, une augmentation de l'exploitation peut, dans un premier temps, provoquer un accroissement de la fertilité des femelles et des animaux récoltés
(Feit 2007 : 68-69). Dans ces conditions, il est inutile de protéger cette ressource, même si, à long terme, le rythme d'exploitation peut influer sur la productivité de la chasse.

Pour de nombreux peuples algonquiens, la pratique régulière de la traite des fourrures a débuté longtemps après l'implantation des Européens en Amérique du Nord. Néanmoins, l'autorité des chefs et les règles de gestion du territoire présentent des similitudes étonnantes avec ce qui a été observé au début du XVII ${ }^{\mathrm{e}}$ siècle. La conclusion que ce mode d'organisation sociale est d'origine précolombienne est presque irrésistible. Par exemple, en Haute-Mauricie, les marchands de fourrures se sont implantés uniquement vers la fin du $\mathrm{XVIII}^{\mathrm{e}}$ siècle. Initialement, le mode de vie des autochtones semble avoir été très peu modifié par leur présence. Vers 1820, on note l'existence de territoires de chasse pour le castor, mais aussi pour l'original. Un chasseur étranger doit obtenir d'un leader une permission pour en exploiter les ressources; ce chef répartit les membres de sa bande sur son territoire (Gélinas 2000 : 46, 91 et 106).

Dans les années 1850, l'augmentation du piégeage des animaux à fourrure paraît due en bonne partie au déclin de la population d'orignaux, qui a vraisemblablement été causé par des feux de forêts (ibid. : 277 et 284). Or, il est clair que cette forme de gestion du territoire a une origine ancienne, alors que la diminution prononcée des castors commence à peine à se faire sentir (ibid. : 158). Ainsi, cette pratique semble bien antérieure au développement des échanges commerciaux dans cette région. Par ailleurs, selon toute vraisemblance, antérieurement aux premiers contacts avec des Européens, même s'ils étaient moins fréquents qu'au $\mathrm{xIX}^{\mathrm{e}}$ et au $\mathrm{xx}^{\mathrm{e}}$ siècle, les feux de forêts ont provoqué des déplacements de population et une pression accrue sur les ressources fauniques qui ont presque forcément suscité l'apparition de territoires familiaux (Feit 2004).

$\mathrm{Au} \mathrm{xx}^{\mathrm{e}}$ siècle dans la région du lac Melville, au Labrador, chez les Innus de Sheshatshit, en l'absence de piégeage du castor, la composition des groupes de chasse, fondée sur la parenté, évolue continuellement; les liens qu'ils entretiennent avec certains secteurs du territoire semblent diffus, sans être inexistants (Mailhot 1985, 1993 : 138-152). Collectivement, ils constituent une bande innue. En général, celle-ci exerce un contrôle territorial dans un bassin hydrographique donné (Mailhot et Vincent 1982 : 71-72). Il en va de même au début du XvII siècle. À cette époque, la bande régionale est d'ailleurs fréquemment qualifiée de «nation».

En définitive, l'importance du désaccord entre Speck et Leacock semble avoir été exagérée. Le premier ne nie pas qu'il existe une différence entre les territoires de piégeage et ceux où l'on chasse le gros gibier, ni que la chasse puisse être communautaire pour certaines espèces et familiale pour d'autres. Tout en admettant que le commerce des fourrures a pu accentuer le phénomène, il soutient que la 
division en territoires familiaux est d'origine précolombienne, ce que semblent admettre bon nombre d'auteurs contemporains. Pour Leacock, il existe des territoires familiaux, mais en l'absence d'un contrôle exclusif et de limites bien définies, elle refuse d'y voir une forme de propriété. Celle-ci n'existerait que dans les territoires de traite qui se seraient développés en raison d'échanges commerciaux. Auparavant, en l'absence d'un risque d'épuisement des ressources, cette délimitation n'avait pas sa raison d'être. De manière similaire, selon Krech, ce sont surtout les Européens qui ont appris aux autochtones à prendre des mesures de conservation.

À notre avis, Leacock a raison de souligner qu'au niveau des groupes de chasse, il n'existe pas d'idée de propriété, mais plutôt des droits d'usage. Elle sous-estime toutefois l'importance et l'ancienneté des limites établies entre les différents districts, ainsi que l'ampleur du contrôle exercé par la nation (ou la bande régionale). À l'inverse, Speck insiste avec raison sur le caractère immémorial des districts, en négligeant la possibilité que les chefs de la nation décident un redéploiement d'un ou de plusieurs groupes en raison des changements démographiques ou socio-économiques. Cela est difficilement compatible avec l'idée de propriété familiale des zones de chasse, bien que, dans ses derniers écrits, Speck semble avoir écarté cette hypothèse pour les périodes reculées. Il faut maintenant voir si cette analyse est confirmée par les sources, en présentant celles-ci de manière exhaustive et détaillée.

\section{LA RECONNAISSANCE DE TERRITOIRES NATIONAUX}

Aux yeux des Français, au début du XviI ${ }^{\mathrm{e}}$ siècle, la nature de l'occupation territoriale des peuples autochtones ne semble pas subir de transformations majeures, contrairement à ce qui se produira dans les décennies suivantes, alors que ceux-ci seront décimés par les épidémies. Les bassins hydrographiques des grands cours d'eaux (fleuve Saguenay, rivières Saint-Jean, Betsiamites, etc.), ou encore les lieux de passage stratégiques comme l'île aux Allumettes dans la rivière des Outaouais, sont occupés par un groupe qui est généralement qualifié de nation, alors que de nos jours les anthropologues parlent plus volontiers de bande régionale. Celle-ci semble dirigée par un capitaine jouissant d'une autorité importante sur les chefs qui dirigent les expéditions de chasse (Morin 2013: 550-561). Les Français sont parfaitement conscients de cette réalité, dont certains documents officiels affirment d'ailleurs l'existence. En outre, les autochtones exercent pendant longtemps un contrôle jaloux sur les voies d'accès aux territoires de leurs voisins. Là encore, les Français comprennent fort bien ces règles, qu'ils cherchent cependant à neutraliser en favorisant la conclusion d'une paix générale entre les nations autochtones. Il faudra attendre près d'un siècle pour qu'une telle paix s'avère durable.

\section{LES RÉFÉRENCES AUX TERRITOIRES AUTOCHTONES}

Dès l'époque de Jacques Cartier, dans la baie de Gaspé, les Stadaconéens font comprendre aux Français qu'ils ne leur permettront pas d'utiliser sans autorisation le territoire où ils se trouvent. En effet, en 1534, après que ceux-ci y ont planté une croix, Cartier raconte qu'un « cappitaine » s'approche avec trois de ses fils et son frère « en faisant le signe de la croix avec deux doydz et puis nous monstroit la terre tout alentour de nous [...] comme s'il eust voullu dire que toute la terre estoit à luy et que nous ne devyons pas planter ladite croix sans son congé » (Cartier, dans Bideaux 1986: 116). Les Français donnent alors à entendre que celle-ci « avoit esté plantée pour faire merche [c.à.d. marque] et balise » afin qu'ils puissent entrer de nouveau dans le havre. Ils leur remettent des présents et s'engagent à revenir l'année prochaine. Leurs interlocuteurs font alors signe « qu'ilz ne habbatroyent ladite croix en nous faisant plusieurs harengues que n'entendions » (ibid. : 117 et 444). Cartier affirme d'ailleurs avoir planté d'autres croix ailleurs pour servir de marques de navigation, sans attacher d'importance particulière à ce fait (ibid. : 131).

Pour sa part, Champlain affirme que les rois de France ont pris possession « d'une bonne partie » des « pays » décrits dans ses ouvrages, tout en reconnaissant que la colonisation a fort peu progressé (Champlain $2010: 11,17)^{2}$. En 1624, Sagard qualifie d'ailleurs la «France Antarctique » de «possession imaginaire en la main d'autruy » (Warwick 1997 : 148). Champlain plante plusieurs croix afin de démentir une telle conclusion, en expliquant aux autochtones que «s'ils les rompaient [...] mal leur arriverait, et les conservant ils ne seraient assaillis de leurs ennemis » (Champlain 2009 : 277). Il ne révèle donc pas que ce symbole pourrait être invoqué contre les puissances européennes qui souhaiteraient établir des liens avec la nation du pays concerné. Toutefois, en réponse aux prétentions que formulent les Anglais sur la côte Atlantique, il attribue une portée fort limitée aux revendications de ce genre :

[...] la rivière qu'ils commençaient à posséder est aux $36^{\mathrm{e}}$ et $37^{\mathrm{e}}$ degrés [...] leur allégation à l'aventure pourrait valoir, s'il n'était question que de tenir cette rivière et sept à huit lieues de l'un et l'autre côté d'icelle, car autant se peut porter la vue pour l'ordinaire. Mais que, s'attribuant par domination, on s'étende trente et six fois plus loin que l'on n'a reconnu, c'est vouloir avoir les bras ou plutôt la connaissance bien monstrueuse. (Champlain 2010 : 196)

Plus généralement, à cette époque, le roi de France revendique à l'encontre des autres puissances européennes un accès exclusif à un territoire où vivent ses alliés, ou encore les peuples qui se sont volontairement assujettis à sa couronne en conservant une autonomie interne presque complète. Toutefois, des revendications de ce genre sont systématiquement rejetées par ceux auxquels elles sont opposées (Morin 2010). Labsence de limites à l'intérieur 
du continent confirme d'ailleurs leur caractère théorique (Greer et Desbarats 2011).

Même lorsqu'il accorde des droits très limités aux autochtones, le Roi reconnaît que ceux-ci étaient jusque-là en possession d'un pays. Ainsi, en 1650, le gouverneur Lauson se fait octroyer la pêcherie d'anguilles sur la pointe de Puiseaux, qui est adjacente aux terres de la mission de Sillery, un peu en amont de Québec, sur le fleuve SaintLaurent. Un jésuite, sans doute Paul Ragueneau, rappelle que « de temps immémorial les sauvages ont eu une entière liberté de faire leur pesche d'anguilles en ceste coste, depuis la pointe de Puiseaux jusqu’à Sillery ». À son avis,

ce seroit une violence extrême, pour ne pas dire une injustice manifeste, d'empescher les sauvages chrestiens de pescher en ce lieu, qui est l'unique qui leur reste, dont ils jouissoient avant que les François fussent jamais venus en ces contrées, dont ils ont jouy paisiblement depuis ce temps-là et sur lequel ils ont fondé leurs espérances, ayans pris dessein de quitter leur vie errante [...] $\mathrm{Ne}$ seroit-ce pas une bien grande cruauté que de pauvres sauvages, en leur propre pays, n'eussent pas aucun lieu pour y chercher leur vie et qu'on les chassast de celuy seul qu'ils se sont réservés (Campeau 1994 : 686-687; voir aussi Lavoie 2010b : 23-31).

En 1651, cette concession est révoquée par la Compagnie des Cent-Associés (Campeau 1996 : 24). Celle-ci accorde le site aux néophytes chrétiens, « considérant qu'il est très raisonnable qu'ils ayent et qu'ils retiennent dans leur paiis l'étendue de terre qui leur sera nécessaire pour vivre en commun et mener une vie sédentaire auprès des François »; bien qu'il ne décrive pas les limites de leur « pays », le document précise qu'ils appellent cet emplacement «Kamik8aa8angachit» (Campeau 1996: 62). La même année, le Roi approuve la concession du fief de Sillery, en précisant que celui-ci est octroyé aux « sauvages qui se retirent ordinairement près de Québec [...] soub la conduite, direction et approbation » des jésuites; l'avis et le consentement de ces derniers sera requis à l'avenir pour « remettre, concéder, vendre » ou « aliéner » ces biens. De la même manière, seul le « capitaine chrestien » pourra " permettre la chasse et la pesche a aucuns particuliers " français, avec la permission « des dits Pères ». Ces derniers se voient aussi confier « la direction des affaires desdits sauvages, sans néantmoins [126] qu'ils soient tenus d'en rendre [compte] qu'à leurs supérieurs » (Campeau 1996 : 61-62). Le 16 novembre 1651, de Lauson interdit d'ailleurs aux Français de contracter directement avec les autochtones (ibid. : 96).

La concession du fief de Sillery ordonne en outre que les « Européans [...] establis dans ces limites [...] demeurent dépendans des capitaines chrestiens et direction des dits Pères » jésuites. En effet, le roi désire « allescher par ces petits émoluments tirés de leur propre païs » les autochtones et les inciter à « quitter leur vie errante et mener une vie chrestienne soubs la conduite de leurs capitaines et desdits Pères qui les ont convertis » (ibid. 62-63).
La mention répétée des capitaines constitue une reconnaissance de leur autorité, aussi bien à l'égard des néophytes que des Européens établis dans leur seigneurie, à l'exception dans ce dernier cas de "la justice », qui devra leur être rendue par les officiers de Québec (ibid. 24, Concession des Cent Associés).

En Nouvelle-France, les représentants du roi admettent d'ailleurs sans difficulté l'autorité des capitaines (Morin 2013). Dans la concession du fief de Sillery, le Roi reconnaît on ne peut plus clairement que les autochtones possèdent le « pays » dans lequel ils chassent et pêchent, même s'il semble présumer qu'ils abandonneront à l'avenir leur mode de vie traditionnel en devenant chrétiens (Campeau 1996 : 791). De la même manière, en 1656, le gouverneur concède des terres aux jésuites, « en tel lieu qu'ils se voudront placer au pays des Irocois supérieurs, dits Onnontagueronons » (ibid.). Bien que ces documents n'aient pas pour objectif de confirmer les droits territoriaux des autochtones, ils reconnaissent l'existence de ceux-ci, antérieurement à l'appropriation française (voir aussi Morin 2010). Juridiquement, celle-ci ne repose pas sur la négation de leurs droits, mais plutôt sur leur élimination graduelle, dans la mesure où cela est nécessaire pour répondre aux besoins de la colonisation. Les régions où les Français ne se sont pas établis constituent donc encore le «pays » des autochtones qui y vivent.

La situation n'a guère changé au $\mathrm{XVIII}^{\mathrm{e}}$ siècle. Les autochtones domiciliés près de Québec et de Trois-Rivières sont encore considérés comme des nations soumises au droit des gens, qui sont tenues d'obtenir la permission des Montagnais avant d'aller chasser sur les terres de ces derniers (Morin 2010 : 42-43). Cette conception du statut des peuples autochtones nous semble avoir pour corollaire la reconnaissance de droits territoriaux, en dépit du discours officiel que tiennent à l'occasion les Français dans le cadre de négociations diplomatiques, lorsque cela favorise leurs intérêts (ibid. : 49-70). Les autorités coloniales protègent d'ailleurs certains de ces droits, même si elles ont le pouvoir de les restreindre unilatéralement en concédant des terres à des particuliers.

$\mathrm{Au}$ minimum, des droits d'usage sont reconnus, notamment dans le Domaine du roi (Lavoie 2010 : 37, 65, 78, 137). Par ailleurs, bien que le traité de paix de 1665 fasse référence à la notion de vasselage, cette terminologie n'a pas été reprise par la suite. On ne saurait donc admettre que, dans l'esprit des parties, «le roi remettait à ses vassaux l'usage des terres sur lesquelles ils chassaient en échange de leur loyauté », ni qu'il leur concédait « un droit d'accès aux ressources » (ibid. : 104-105). Au contraire, les autochtones étaient considérés soient comme des alliés, soit comme des sujets du roi possédant leurs propres territoires depuis des temps immémoriaux; en effet, les sources font régulièrement référence aux « pays» ou aux « terres » 
où ils chassent (Morin 2010; infra, « Le contrôle de l'accès au territoire $»$ ).

Les observations d'explorateurs et de missionnaires confirment cela. À de très nombreuses reprises, les autochtones fournissent une description détaillée de régions et de populations plus ou moins éloignées, parfois avec une représentation schématique tracée sur le sol ou sur un morceau d'écorce. Selon Lahontan, les autochtones conservent d'ailleurs un grand nombre de cartes d'écorce (Lahontan 1990a : 645). Ces renseignements concordent généralement avec les connaissances actuelles (Bideaux 1986 : 132, 156, 168, 199-200; Champlain 2009 : 33, 43, $108,164-165,190,198,238,262,274-276$ et 324 ; Warkentin 2012 : 287). En outre, on trouve fréquemment des expressions telles que la « demeure », les «terres » ou le «pays » d'une nation autochtone (Champlain 2009 : $37,40,42,48,187,202,215,271,299)$. En 1616, le père Biard affirme qu'en Nouvelle-France, « chasque peuple a sa langue et sa contrée à part » (Campeau 1967 : 478). Par ailleurs, les voies de navigation sont complétées par un grand nombre de portages qui permettent de parcourir d'immenses distances, par exemple de la baie de Fundy au Saint-Laurent (Lescarbot 1610: 40; Denys: 473-474, 496, 503; Ganong 1908 : 473-474, 496, 503).

En revanche, Champlain écrit souvent que les endroits où se pratiquent la chasse et la pêche sont « habités de sauvages qui vivent errants parmi le pays », ou quelque chose d'analogue (ibid. : 165, 190 267, 296-297, 329; voir aussi Champlain 2010: 76). Cette fréquentation saisonnière ne présente guère d'intérêt pour lui, d'autant qu'il parcourt de grandes distances sans rencontrer âme qui vive (voir Champlain 2009: 43, 85, 92-93; Campeau 1967: 476). Bien qu'il fasse la promotion d'une colonisation pacifique à des fins de christianisation, Marc Lescarbot, qui est avocat de Paris, affirme de but en blanc: "La terre donc appartenant de droit divin aux enfans de Dieu, il n'est ici question de recevoir le droit des Gens, \& politique, par lequel ne seroit loisble d'vsvrper la terre d'autrui »; toutefois, « il la faut posseder en conservant ses naturels habitans » (Lescarbot 1907 : 219). Lescarbot est néanmoins très conscient du fait que ceux-ci occupent des régions bien déterminées (Lescarbot 2007 : 133, 218). À cet égard, la position française a peu varié : l'occupation du territoire par les autochtones n'impose aucune obligation à la Couronne française dans le cadre de la colonisation. Toutefois, dans les régions où il n'a pas été exercé, le simple pouvoir de concéder des titres fonciers ne fait pas disparaître leurs droits.

Même si cela intéresse rarement les explorateurs, les autochtones peuvent décrire avec précision les limites de leur territoire. Ainsi, en 1536, Cartier apprend que la " terre et prouynce de Canada » débute à environ sept lieues de l'île aux Coudres, là où se trouvent « quatorze » îles du fleuve Saint-Laurent, notamment l'île aux Grues (Bideaux 1986 : 136). De son côté, Roberval note que les Iroquoiens du Saint-Laurent « ont un roi dans chaque province auquel ils obéissent de façon étonnamment soumise » (ibid. : 209). Précisons qu'à compter du début du $\mathrm{XVII}^{\mathrm{e}}$ siècle, sous l'influence de Lescarbot, ce terme sera très vite remplacé par celui de "capitaine», afin de mieux rendre compte de la nature limitée des pouvoirs détenus par ceux-ci (Cook 2007).

Plusieurs observateurs signalent l'existence de limites territoriales bien définies. En 1615, près du lac Huron, Champlain rencontre trois cents Cheveux-Relevés [ou Outaouais] et s'entretient avec leur chef « sur ce qui était de son pays, qu'il [...] figura avec du charbon sur une écorce d'arbre » (Champlain 2009 : 299). Un chef non identifié rappelle également à Chestien Leclerq « que la Nation des Gaspesiens s'étend depuis le Cap des Rosiers, jusqu'au Cap Breton ». En revanche, les « Sauvages de Restigouche » vivent dans la Baie des Chaleurs; ces «frères » et « compatriotes », qui parlent la même langue qu'eux, ont été baptisés par d'autres missionnaires (Leclercq 1999 : 391). Le terme «frère » désigne fréquemment les membres d'une même nation (Morin 2013); dans cet exemple, comme dans d'autres documents, la nation a une portée beaucoup plus large que la simple bande occupant un bassin hydrographique.

Sagard mentionne au passage la « frontière, pour entrer és terres ennemies » lorsque l'on quitte le pays des Hurons (Warwick 1997 : 235, 238). Précisons toutefois que, dans son esprit, les «frontières » semblent passer par les champs cultivés; il s'agit peut-être de la lisière de la forêt (ibid : 244). Tout comme Lescarbot, il affirme que la guerre n'a jamais pour objectif de "posséder les terres et conquerir » un « pays » (Warwick 1997 : 244; Lescarbot 2007 : 444; voir aussi Boucher 1964 : 117; Leclercq 1999 : 509). En effet, elle semble principalement motivée par la capture des captifs destinés à compenser les pertes de population (Brandao 1997; Viau 1997; Havard 2003; Parmenter 2010). Par ailleurs, on peut parfois découvrir « certaines marques que les chefs se donnent d'une nation à l'autre, s'avertissant de temps en temps quant ils en changent, et par ce moyen ils reconnaissent [si] ce sont amis ou ennemis qui ont passé » sur les terres d'une nation (Champlain 2009 : 195; Warwick 1997 : 323 ; Leclercq 1999 : 392). Ainsi, en 1666, les Papinachois quittent Tadoussac pour rentrer chez eux; ils peignent des marques distinctives sur certains troncs d'arbres, afin d'indiquer qu'ils sont passés par là (JR, 50 : 20). En général, celles-ci ne semblent pas destinées à fixer des limites; elles signalent plutôt les déplacements récents, soit pour rassurer la nation qui se considère maître de la région, soit en guise de défi.

Même s'ils doivent parfois se résigner à le quitter, les autochtones demeurent attachés à leur pays. Ainsi, en 1644, 
selon Barthelemy Vimont, les Algonquins « ont esté chassé de leur propre pays » par les Iroquois, parce que « les bois les plus commodes à la chasse » s'y trouvent, de chaque côté du Saint-Laurent (Campeau 1990b : 59, 223). En 1646, ils réintègrent temporairement leur territoire (Campeau 1996 : 75). En 1647, trois autochtones de la Petite Nation des Algonquins ont également « resolu de quitter leur paiis $»$ en raison des attaques iroquoises, sans compter qu'une épidémie décime les caribous (Campeau 1994: 91). En 1660, dans la région située au nord du lac Saint-Jean, trois nations se réfugient aussi vers l'est en raison des raids iroquois (Campeau 2003 : 471). En 1668, selon un missionnaire, l'ancienne demeure des Outaouais était « vn quartier $\mathrm{du}$ Lac des Hurons d'où la crainte des Iroquois les a chassez, \& où se portent tous leurs desirs comme à leur pais natal » (JR, $51: 20)$. Ces affirmations signifient simplement que la guerre menée par les Iroquois a fait fuir leurs ennemis; avant le dernier tiers du XVII ${ }^{\mathrm{e}}$ siècle, l'accès aux zones de chasse ne semble pas avoir été une motivation principale des conflits (Brandao 1997; Viau 1997; Havard 2003; Parmenter 2010).

À la Baie d'Hudson, en 1672, les nations des Kinistinons, des Mataoüakirinoüeks \& Monsouniks sont séparées chacune « par de grandes rivières »; elles ont donc leur territoire distinct (JR, 56: 202). D'autre part, Lescarbot et Champlain signalent souvent le nom du « chef » ou du « capitaine » d'une rivière et des autochtones qui y vivent (Lescarbot 2007: 186, 216-217; Champlain 2009: 91, 102, 132; 2010 : 38). De même, le père Buteux parle du lac où son hôte «fait sa demeure la plus ordinaire » (Campeau 1996 : 137). En 1646, à l'occasion d'une paix conclue entre les Betsiamites et les autochtones d' « Acadie », on apprend que les régions de Tadoussac, de la baie des Chaleurs, de l'Acadie et de la baie de Rigibouctou ont chacune leur capitaine (Campeau 1990b : 691-693). En 1652, on signale à nouveau des hostilités féroces entre les « sauvages de Gaspé » et les « Oumiouek ou Bersiamites » de la Côte-Nord (Campeau 1996 : 313; voir aussi Leclercq 1999: 510). Il en va de même au Lac Manicouagan (JR, 49 : 52).

$\mathrm{Au}$ début du $\mathrm{xvIII}^{\mathrm{e}}$ siècle, Lahontan écrit que les autochtones « se font la guerre au sujet de la Chasse ou du passage sur leurs terres, parce que les limites sont réglées » et que chaque "Nation connoît les bornes de son Païs " (Lahontan 1990a: 712). Selon lui, en 1684, les cinq nations iroquoises reprochent aux Illinois et aux Oumamis de la région des Grands Lacs d'avoir envahi leurs territoires de chasse, en plus de violer une règle universellement admise, selon laquelle il faut laisser la vie sauve à certains castors, sans aucun doute afin qu'ils se reproduisent. En agissant ainsi, ces nations

[...] ont coupé les Arbres de Paix qui servoient de limites à nos Frontiéres. Ils sont venus faire des grandes chasses de Castors sur nos terres, ils en ont entiérement enlevé \& masles \& femelles, contre la coutusme de tous les Sauvages. [...] Nous avons moins fait que les Anglois \& les François, qui sans droit ont usurpé les terres qu'ils possedent sur plusieurs Nations qu'ils ont chassées de leur païs pour bâtir des Villes, des Villages \& des Forteresses. (ibid. : 308-309)

Les cinq nations affirment également avoir chassé aux environs du fort Frontenac (devenu Kingston) « depuis que le monde est monde » (La Potherie 1997a : 655).

En 1697, dans la région du fort Nelson sur la rive occidentale de la baie d'Hudson, selon Bacqueville de La Potherie, la plupart des guerres entre nations sont dues aux conflits concernant les « droits de chasse » (1997a : 105). À son avis, il en va de même « de toutes les guerres entre les Iroquois et tous nos alliés, parce que les Iroquois sont fort bornés » et parce qu'il y a " très peu de Pelleterie » dans les régions où ils vivent, ce qui les pousse à attaquer leurs voisins (La Potherie 1997b: 447). Tous ces documents prouvent que les nations autochtones connaissent les limites de leurs territoires et entendent les faire respecter. Les Français sont bien conscients de cette réalité. De même, ils ont rapidement compris le rôle primordial joué par les capitaines régionaux dans le contrôle de l'accès aux territoires de leur nation.

\section{LE CONTRÔLE DE L'ACCÈS AU TERRITOIRE}

Dès 1535, à Stadaconé (devenu Québec), le chef Donnacona s'oppose au projet de Cartier de se rendre sans son consentement à Hochelaga, sur l'île de Montréal (Bideaux 1986 : 142-143, 145). Champlain est également contraint de renoncer à son idée d'explorer le Saguenay ou le Saint-Maurice, en raison du refus de ses alliés de les y conduire (Champlain 2009 : 165, 208, 230). D'autres peuples tentent avec insistance de le dissuader de poursuivre son exploration vers l'ouest, afin de préserver leur position d'intermédiaire (ibid. : 265, 272-274; Warwick 1997 : 176). Pour la même raison, en 1642, les Montagnais de Tadoussac ne veulent pas que Paul Le Jeune les accompagne dans leur expédition de traite avec des «nations plus éloignées » (Campeau 1990a: 153-154). En 1659, l'accès au Saguenay dépend toujours de la volonté de certains peuples (Warkentin 2012: 245). Par ailleurs, Champlain est bien conscient du fait qu'il faut offrir des présents pour pouvoir traverser un territoire de manière sécuritaire. En effet, en 1611, il promet à ses alliés de revenir dans un an avec une cinquantaine de soldats. Si ceux-ci lui fournissent les vivres et le transport, il s'engage à apporter « de quoi faire des présents aux chefs qui sont dans les pays » où ils passeront (Champlain 2009: 239; voir aussi Warwick 1997 : 188).

Les autochtones n'hésitent pas à imposer le respect de cette coutume. En 1610, Lescarbot rapporte que les Micmacs prennent ce qui leur plaît dans un navire de pêcheurs français qui a jeté l'ancre près de leurs « cabanes » et qu'ils font provision de poissons frais au retour des chaloupes (Lescarbot 2007: 225). En 1624, à Tadoussac, le chef 
Erouachy (connu aussi sous le nom de La Forière) est insulté du petit présent qui lui été offert par un capitaine français. Il aborde alors l'embarcation de celui-ci, où ne se trouvent que six ou sept personnes, en invitant ses compagnons à se servir librement et à donner ce qu'ils veulent pour les marchandises. Le soir venu, ils se ravisent et offrent plus de pelleteries qu'il n'en faut pour compenser la perte encourue (Warwick 1997 : 141). Le Jeune observe d'ailleurs que les Montagnais « n'ouvrent point la main à demy, quand ils donnent [...] entre eux», mais qu'« ils sont ingrats au possible envers les estrangers » (Campeau 1979 : 600) - alors que Leclercq fait la même observation pour les Micmacs (1999 : 371).

En 1633, Champlain demande au "capitaine » des Montagnais de Québec d'empêcher ses hommes d'aller trafiquer avec des contrebandiers anglais qui les attendent en aval sur le fleuve. Il menace de dépêcher des embarcations armées et d' « user de la coustume qu'ils ont entr'eux, qui est que touttes personnes qui vont traitter avec leurs ennemis, on leur saisit leurs marchandises et leurs commoditez, sans les laisser passer » (Campeau 1979: 365). En 1623, à Québec, lors d'une assemblée des différentes nations alliées, les Hurons se plaignent que les « passages [...] n'étaient pas libres », parce que «les Algonquins les traitaient mal, leur faisant contribuer de leurs marchandises $»$ ou les pillant purement et simplement. Un compromis et une forme de dédommagement sont alors trouvés avec cette nation, qui pourrait être celle de l'île aux Allumettes (Champlain 2010: 51, notamment n. 39). Le problème se pose de nouveau en 1625; le frère Gabriel Sagard, en tant que Français, réussit à éviter ces mauvais traitements à ses compagnons de voyage, car en lui bloquant le passage, les Algonquins de l'île craignent de s'exposer à des représailles lorsqu'ils se rendront à Québec pour la traite (Warwick 1997 : 326-328).

Peu après, à la hauteur du Richelieu, les Montagnais et les Algonquins de la région de Trois-Rivières veulent empêcher les Hurons de se rendre à Québec. Ces derniers offrent des présents à leur vis-à-vis, mais Sagard convainc ses compagnons de ne pas suivre cet exemple (ibid. : 334335). Il prend la fuite avec eux de bon matin, mais à une ou deux lieues de Québec, il est arrêté par le «Capitaine » d'un « village de Montagnais dressé sur le bord de la rivière », avec « plusieurs autres de sa bande ». Celui-ci exige du blé d'Inde et de la farine « comme estan deu (disaient-ils) à leur Capitaine, pour le passage et entrée dans leurs terres » (ibid. : 337). Une embarcation française envoyée "pour empescher leurs insolences » leur fait lâcher prise. Il semble donc que les Montagnais de la région de Québec perdent à cette époque le contrôle sur la circulation fluviale, mais il serait plus exact de dire qu'ils font une exception, à leur corps défendant. Comme l'établissement de monopoles commerciaux, ces modifications marquent le début d'une domination française dans l'estuaire du Saint-Laurent. Elles correspondent à un « effritement » de la souveraineté des autochtones (Beaulieu 2008). Ainsi, les capitaines régionaux vivant à proximité des Français ne pourront plus contrôler la circulation à l'intérieur de leur territoire régional.

En 1633, alors que Champlain détient un Algonquin de l'île qui a reconnu avoir tué un Français, les Hurons refusent d'embarquer des jésuites, car le bruit court que les parents du meurtrier attaqueront ces derniers en guise de représailles. Ils expliquent que « la rivière n'estoit pas à eux et qu'on prit garde avec ces autres nations s'ils ne pourroient passer avec asseurance; que pour eux, ils ne demandaient pas mieux que d'embarquer des François » (Campeau 1979 : 479). Les missionnaires renoncent à leur projet, de peur de provoquer «une guerre contre ces peuples », car plusieurs nations voient d'un très mauvais œil le développement des relations entre les Hurons et les Français. De son côté, Brébeuf explique à ses "frères » hurons que, "puisque la rivière est bouchée ", ses collègues et lui attendront l'année suivante pour se rendre dans leur pays (Campeau 1979: 483; pour d'autres exemples, voir Campeau 1987: 85-86, 331). Pour leur part, les Ouperigoues Ouaouakhis, qui vivent à l'intérieur des terres sur la Basse-Côte-Nord, n'ont jamais rencontré d'Européens, mais ils n'osent venir à Tadoussac pour y commercer. En effet, ils craignent d'être tués par les Montagnais de cette région. En 1635, ceux-ci ont d'ailleurs abattu impitoyablement des Bersiamites qu'ils avaient pourtant accueillis pacifiquement parmi eux (Campeau 1987 : 73-74). Le Jeune fait un rapprochement entre la crainte des Ouperigoues Ouaouakhis et l'exécution des Bersiamites, même s'il ne connaît pas réellement les causes de ce dernier drame.

Avant d'entreprendre le voyage vers Québec, les Hurons s'assurent toujours que les Algonquins de l'île leur laissent le «passage libre » (Campeau 1987 : 288). Bien qu'ils soient dix fois plus nombreux que ces derniers, ils « ne passeront pas, si vn seul Insulaire s'y oppose, tant ils gardent étroittement les loix du Païs »; toutefois, les « presens ouurent pour l'ordinaire cette porte, quelque fois on les fait plus grands, quelquefois plus petits, selon les occurrences » (ibid. : 297-298). Au décès d'un capitaine, il faut offrir plus pour la cérémonie de transmission du titre et du nom du défunt, au cours de laquelle on remettra des cadeaux à la famille de celui-ci; on dit alors qu'il est « ressuscité » (voir Savard 1996; Morin 2013 : 559-560). Par la suite, « on ne paye que le tribut ordinaire quand on passe sur les marches, \& sur les limites de ces Insulaires » (Campeau 1987 : 298; voir aussi Campeau 1990a : 475). Précisons que le terme "marches » désigne «Toute province, région située en bordure d'un pays étranger ou d'une province voisine » (Centre national de ressources textuelles et lexicales, <http://www.cnrtl.fr/definition/marches $>$ )

En 1637, des Abénaquis vivant dans l'actuel État du Maine veulent se rendre à Trois-Rivières pour y trafiquer 
avec des Algonquins, mais le « Capitaine » des Montagnais de Québec leur défend de le faire. Il demande alors l'appui du gouverneur afin de «fermer tous les fleuues par où ils peuuent retourner en leur paiss (Campeau 1987 : 658). Le gouverneur interdit alors au capitaine des Abénaquis d'aller à Trois-Rivières, mais la bande passe outre. Interrogés par la suite, ils disent vouloir uniquement secourir leurs alliés. De fait, les Français fouillent leur cabane et ne trouvent aucun castor; ils confisquent cependant les trois arquebuses que les Abénaquis ont en leur possession. Le Jeune explique alors la "façon de faire de ces nations » lorsqu'elles décident de « boucher le passage » :

Ces Barbares ont vne coustume assez remarquable quand quelques autres nations arriuent en leur pays, elles n'oseroient passer outre sans la permission du Capitaine du lieu, autrement on briseroit leurs canots; cette permissiō de passer se demāde les presens en la main: si le Capitaine n'agrée pas leur presens, n'aiant pas enuie de les laisser passer, il leur dit qu'il a bouché les chemins, \& qu'ils ne sçauroient passer. A ces parolles il faut rebrousser chemin ou se mettre en danger de guerre. (ibid. : 659)

Il est remarquable de constater que le capitaine des Montagnais de Québec estime qu'il détient ce pouvoir sur le fleuve Saint-Laurent, même s'il demande l'appui des Français avant de l'exercer. Vers 1660, Pierre-Esprit Radisson est également bien conscient de la nécessité d'offrir un présent aux Nadoueseronons afin que ceux-ci laissent le « passage libre » à son groupe (Warkentin 2012 : 280). Pour leur part, en 1668, en amont de Montréal, les Outaouais prétendent que le fleuve Saint-Laurent «leur appartient, \& qu'aucune nation n'y peut nauiger, [81] sans leur consentement $»(J R, 51: 20)$, ce que les Iroquois ne sont certes pas disposés à admettre (Parmenter : 133 et suiv.). $\mathrm{Au} \mathrm{XVIII}{ }^{\mathrm{e}}$ siècle, les peuples qui vivent dans la région $\mathrm{du}$ fort Nelson s'expriment de manière semblable (La Potherie 1997a : 126)

Selon Pierre-Esprit Radisson, les Iroquois ont pour coutume de dépouiller de tous ses biens une personne perdue dans les bois (Warkentin 2012: 196) ${ }^{3}$. Chez les Montagnais, le pillage des biens constitue également une sanction entre membres d'une nation, par exemple lorsqu'une veuve se remarie moins de trois ans après le décès de son défunt mari. Son nouveau conjoint risque d'être dépouillé de tous ses biens par l'ancienne belle-famille de son épouse, parce qu'il n'a pas respecté le délai de viduité. Le Jeune précise: " cette coustume est tellement passée pour loy que nous l'avons veu prattiquer devant nos yeux, en sorte que celuy qui s'estoit ainsi marié veut prendre ses colliers de porcelaine et tout ce qu'il avoit, sans dire autre chose, sinon que c'estoit luy qui se faisoit ce tort, pour avoir enfreint leur coustume » (Campeau 1989 : 346, 1990a: 474). En cas de vol, les autochtones vont également « dépouiller les premiers qu'on rencontre de la parenté ou de la nation de celuy qui a [23] fait le vol» (Campeau 1996: 290). Cette règle constitue une reconnaissance très claire de l'importance pour les autochtones de leurs biens personnels, puisqu'ils peuvent en être privés afin de sanctionner une transgression.

En 1638, Jérôme Lalemant voyage avec des Hurons en direction de leur pays. Sur la rivière des Outaouais, ils rencontrent quatre "cabanes d'Algonquins de l'Isle». L'un d'eux est outré parce que quelques jours plus tôt, un Français est passé par là et a saigné un malade, qui est décédé peu après. Il met alors une corde autour du cou de Lalemant et se saisit d'une hache dans le dessein de le faire mourir parce qu'il appartient à la même nation que l'apprenti médecin. Les Hurons interviennent en sa faveur et obtiennent sa libération à force de présents. À la fin, l'Algonquin déclare qu'il n'est plus fâché. Lalemant ajoute que le lendemain, un Huron a bien failli tuer l'agresseur sur-le-champ. Ses compagnons demandent que l'incident soit rapporté au gouverneur. Lannée suivante, le même Algonquin se retrouve à Trois-Rivières avec un capitaine de sa nation. Ce dernier se remet peu à peu du coup de hache sur la tête que lui a assené un troisième Algonquin, auquel il a généreusement pardonné. Les Français veulent alors obtenir satisfaction pour le mauvais traitement infligé au père Lalemant l'année précédente. Le chef s'« estonne que ceux qui font estat de prier Dieu et qui [160] disent qu'il faut pardonner les offences, puisque Dieu les pardonne, veuillent tirer vengeance d'une injure qu'on leur a fait, il y a desjà longtemps ». Laffaire en reste là (Campeau 1989 : 349).

Peu avant son décès survenu en 1717, Nicolas Perrot donne une tout autre interprétation de ces événements, qu'il situe en 1650. Selon lui, en abandonnant le pays des Hurons pour se réfugier à Québec avec ses néophytes, Lalemant a refusé de se rendre au village des Algonquins de lî̀le aux Allumettes, où le chef Le Borgne exigeait un péage. Il aurait déclaré que «le François estant le maître de la terre, n'estoit point obligé à cela » (Perrot 2007 : 136). Le Borgne les ayant découverts, les Hurons rejetèrent le blâme sur le père Lalemant. Il fit suspendre celui-ci « à un arbre par les aisselles, en luy disant que le François n'estoit pas maistre de son pays; qu'il en estoit luy seul reconnu pour chef, et qu'on y estoit sous sa puissance ». Descendu à Québec l'année suivante, il aurait été enfermé dans un cachot et libéré quelques jours plus tard, après que ses compagnons eurent offert des dons aux Français (ibid.). Cette version n'est attestée par aucune autre source; en outre, elle souffre de plusieurs lacunes qui la rendent très peu plausible. À ce sujet, Lucien Campeau écrit :

Cet épisode sera déformé par la tradition orale rapportée par un Algonquin au Sault-Sainte-Marie à Perrot, vers 1670. Le nom de Lalemant est exact, mais pas la date (1650), la destination (descente vers Montréal au lieu d'une montée), le lieu (île aux Allumettes plutôt qu'un point en aval), le motif (péage au lieu du deuil) et les conséquences (emprisonnement du Borgne au lieu des reproches de Montmagny). (Campeau 1989: 129-130, n. 7).

Ainsi, en 1643, Ag8achimagan, capitaine des Algonquins de l'île, déclare que ceux-ci ont été décimés par la maladie, 
la guerre et la famine, de telle sorte qu'ils sont « réduits au néant » (Campeau 1990b : 215). Il est donc peu probable que les survivants aient pu exercer un contrôle sur la circulation dans cette région. Pourtant, la description de Perrot est parfois présentée comme faisant autorité (D'Avignon 2008 : 89-90).

En 1672, le père Charles Albanel et son groupe entrent sur les terres de la Nation des «Mistassirinins »; Moukoutagan, qui est membre de celle-ci, lui demande de s'arrêter là, afin qu'il avertisse de leur arrivée un « vieillard, maistre de ce païs $»(J R, 56: 172-174)$. Sachant que les autochtones « sont extremement reservez à donner passage aux estrangers, pour aller aux Nations éloignées », Albanel explique au capitaine de cette région :

\begin{abstract}
Sesibahoura, ce n'est pas pour achepter le passage de cette riviere, \& de ton Lac, que je te veux regaler de deux presens. Le François ayant delivré tout ce païs des incursions des Iroquois vos ennemis, merite bien qu'on luy fasse un droit d'aller, \& de venir avec toute liberté sur cette terre, qu'il a conquise par ses armes. De plus, Dieu, que vous distes vous [173] mesmes estre le maistre de toutes choses [...] me donne le droit de passer librement par tout. L'Annié, I'Oneiout, l'Onnatagueronon, l'Oiogouen, ny le Sonnotouan, le Nepissirinin, l'Outaouac, ny toutes les Nations estrangeres n'ont jamais rien exigé de mes freres, lorsqu'ils passent \& repassent librement sur leurs terres pour les instruire, \& les informer des Loix de l'Evangile. (ibid. : 170)
\end{abstract}

Deux prétentions différentes sont présentes ici. D’une part, en tant que membre de la nation qui a imposé la paix aux Iroquois, un Français « mérite bien » de circuler librement partout, y compris dans les régions proches de la baie James, qui auraient été à son avis conquises par les armes (sans qu'un seul soldat français n'y ait jamais mis les pieds). D'autre part, les religieux ont toujours considéré qu'ils avaient le droit de circuler librement pour prêcher l'évangile. Le lendemain, le capitaine exprime sa gratitude aux Français, qui leur ont donné la paix; il demande ensuite au père Albanel de demeurer parmi les siens pour les instruire ou les baptiser, ce que ce dernier accepte de faire pour quelques jours (JR, 56: 176-178). Lannée suivante, des Mistassins se rendent à Québec pour demander la protection du gouverneur contre les Iroquois, "l'assurer qu'ils le prenoient pour leur père », en promettant qu'ils continueront de prier (JR, 59: 44); cela n'implique d'ailleurs aucune forme de soumission (Morin 2013 : 580-587). Albanel semble penser qu'il a imposé « la libre circulation des sujets » français - un point de vue adopté par Dawson (2011: 107). À notre avis, il a simplement obtenu la permission de continuer son chemin. Pour le reste, beaucoup de questions demeurent en suspens à la suite de cette rencontre.

Pour en revenir à l'expédition de 1672, arrivés à la Baie James, ses membres finissent par rencontrer le capitaine Kiaskou. Albanel explique qu'Onnontio, qui a fait la paix avec les Iroquois, rend son pays à ce capitaine, afin que celui-ci puisse pêcher, chasser et trafiquer en toute sécurité. Il assure que les Français viennent dans ces régions uniquement pour convertir les autochtones, plutôt que pour y commercer (JR, 56 : 192). Le capitaine s'inquiète de voir qu'Albanel n'est pas en état de rapporter des présents au gouverneur : « [...] on dira à Québec, que ie n'ay point de bouche, que je suis un enfant, qui ne sçais pas parler » (ibid. : 196). Dans sa réponse, Albanel tente à nouveau de faire reconnaître le droit des Français de circuler partout sur le territoire, mais il est contraint de se rabattre sur son statut de religieux et de laisser non résolue la question des expéditions à des fins commerciales. Rien n'indique que ses interlocuteurs ont accepté de renoncer à leur contrôle territorial, même s'ils souhaitent volontiers devenir les alliés des Français.

En 1684, selon Radisson, aux environs de la rivière Nelson et des rives occidentales de la baie d'Hudson, les autochtones ont accepté l'abolition de la «coutume» selon laquelle il était nécessaire d'offrir des présents pour qu'ils acceptent de changer de partenaire commercial (Radisson 1999 : 341). Son neveu Jean-Baptiste Desgroseillers constate d'ailleurs l'importance de cette règle. Chargé de la garde du fort français construit en 1682, il rencontre un groupe d'autochtones de la rivière New Severn, située à quelques centaines de kilomètres en direction sud-est. Ceux-ci s'en vont traiter avec les Anglais, plus exactement avec leurs «frères établis au fond de la baie », plus au sud. Des Groseillers les invite à venir fumer du tabac avec les Français, ce qui est synonyme de paix (Radisson 1999 : 347-348). Il est alors victime d'une tentative de meurtre. Son agresseur, "qui était le chef de toutes les nations et l'ami des Anglais du fond de la baie », lui reproche ne pas avoir « payé par présent la possession du pays » où il s'est établi (ibid. : 348). Ce sont d'ailleurs les Anglais qui l'ont incité à agir ainsi, lors d'une rencontre secrète tenue peu de temps auparavant.

Plusieurs autochtones décèdent au cours des affrontements qui résultent de cette agression, dont certains sont parents de celui qui était devenu le père adoptif de Radisson (ibid. : 349-361). Afin d'éviter un conflit, ce dernier organise une rencontre avec le gouverneur de la Compagnie de la Baie d'Hudson, pour le compte de laquelle il travaille désormais. Il explique qu'il convient de faire «quelques petits présents » au chef de la nation du « lieu » où la compagnie a bâti son fort; ce personnage n'est autre que son père adoptif (Radisson 1999: 361). Le gouverneur s'emporte et refuse. De son côté, Radisson a refusé d'offrir des présents « à un simple Sauvage » qui « servait d'espion » au gouverneur et qui était le fils d'un chef régional vivant en amont de la rivière New Severn (ibid.).

Radisson affirme qu'il «n'a jamais fait de présents qu'aux chefs de ces nations » (ibid.). Il souligne les « inconvénients » pouvant découler d'une « indifférence » 
envers ceux-ci, surtout là où la compagnie prétend s'« établir ( (ibid. : 362). En réalité, il n'a jamais reconnu l'autorité du chef qui semble avoir été l'interlocuteur des Anglais, car il avait noué l'année précédente une alliance avec son "père adoptif », alors qu'il travaillait pour les Français. Nous n'avons que sa parole quant à l'autorité de ce dernier; il semble cohérent dans ses récits, même s'il mentionne au passage la présence d'un autre « grand chef et capitaine des Sauvages » dans la région (1999: 345; voir Promislow 2008 : 55, 2010 : 77). Quoi qu'il en soit, il est clair que ces chefs revendiquent le droit de décider quels Européens pourront s'établir parmi eux et qu'ils s'attendent à recevoir des présents en contrepartie de leur autorisation. En outre, l'un d'entre eux réside à une grande distance, soit aux environs de la rivière New Severn, ce qui indique qu'il prétend contrôler une vaste région.

Dans l'ensemble, le contrôle de l'accès au territoire par des chefs régionaux est attesté sur la côte Atlantique, dans la vallée du Saint-Laurent et dans la région de la baie d'Hudson. Les autorités coloniales ont dû s'assurer qu'il ne nuise pas au commerce des fourrures, parfois en imposant graduellement leur autorité (comme sur le Saint-Laurent, en aval de Montréal), parfois en favorisant des accords avec les peuples autochtones.

\section{LA MISE EN COMMUN DES RESSOURCES}

Tout au long du XVII e siècle, les Français feront beaucoup d'efforts pour obtenir la conclusion d'une paix générale fondée sur l'établissement de relations fraternelles et la mise en commun des zones de chasse (Morin 2013 : 587-596). Ils sont bien conscients du fait que chaque nation surveille jalousement les déplacements à travers son territoire. Dans la vallée du Saint-Laurent, l'accès aux régions giboyeuses intéresse particulièrement les Iroquois, tandis que leur hostilité paralyse en pratique les alliés des Français, qui ont de plus en plus de difficulté à transporter leurs fourrures (Champlain 2010 : 42, 93 et 95; voir aussi Parmenter 2010). Dès 1603, Champlain réalise l'intérêt d'amener toutes ces nations à faire la paix; par la suite, il soutiendra ce projet de toutes ses forces, mais les hostilités reprendront assez vite (Champlain 2009 : 30, 2010 : 40-41, $90,122)$. Les négociations de ce genre sont d'ailleurs assez délicates. En 1644, un autochtone estime que la construction de forts français a pour effet de «boucher» les rivières pour les Iroquois et de permettre aux chrétiens de « vivre comme frère $[s]$ » sans se quereller, bien que cela soit « difficile, quand il s'agit de traite » (Campeau 1990b: 143). Réciproquement, du point de vue des Iroquois, advenant la fin des hostilités, la « rivière sera toute calme et toute unie et la paix [...]» fera que les anciens belligérants seront « tous amys » (ibid. : 361-362).

En 1645, dans le cadre de pourparlers de paix, l'« ambassadeur » Agnier Kiotseaeton offre à ses hôtes autochtones de « venir manger avec eux », car son pays
« est remply de poisson, de venaison, de chasse » et «plein de cerfs, d'élans, de castors » (ibid. : 369). Cette même année, après la conclusion définitive de ce traité avec les Montagnais, les Algonquins et les Hurons, un ambassadeur iroquois invite ses interlocuteurs à se rendre dans le pays qui les sépare, lequel est « rempli d'ours, de cerfs, d'élans, de castors et de quantité d'autres bestes » (ibid. : 379). Par la suite, les Agniers viennent chasser en grand nombre " dans les confins des Algonquins », qui les reçoivent pacifiquement dans leur habitation chrétienne (ibid. : 551). Selon Jérôme Lalemant, comme « la pluspart des animaux » se trouvent « sur les marches des Algonquins », les Agniers profitent de la paix pour « en tirer à leur aise et sans crainte ", avec " passion », c'est-à-dire qu'ils abattent un grand nombre de bêtes (ibid. : 554). Les hostilités reprennent toutefois l'année suivante (voir notamment Morin 2013).

En 1653, lors de nouveaux pourparlers, un Agnier demande qu'après la conclusion de la paix « la chasse fût commune entre toutes les nations confédérées et qu'on ne fist plus la guerre qu'aux élans, aux castors, aux ours et aux cerfs, pour gouster tous ensemble les frians mets qu'on tire de ces bons animaux »; de cette manière, sa nation pourra se procurer des castors (Campeau 1996: 605). Selon des "ambassadeurs » abénaquis, après cette paix, « on ne craindra plus les embuscades » et « tous ceux qu'on rencontrera seront autant d'amis » (ibid. : 611). Leur porte-parole ajoute: " mes frères [...] allons de compagnie à la chasse de l'élan et du castor » (ibid. : 612). La même idée est à la base du traité de 1654 entre les Iroquois, les Français et leurs alliés (ibid. : 695). Elle est traduite de manière plus imagée par le père Ragueneau. Celui-ci invite les Onéïouts « à aller à la chasse sans crainte en quelque endroit que ce soit et que s'ils rencontroient les Algonquins, ils se fassent chaudière et se donnent de la viande ", c'est-à-dire qu'ils fassent cuire leur nourriture dans une petite marmite et la partagent entre eux (ibid. : 903). Les Agniers procèdent de la même manière lorsqu'ils rencontrent un groupe de Français en forêt (Campeau 2003 : 59). En 1658, lors de pourparlers de paix, les Onontagués demandent à nouveau la liberté de chasser pour tous les Iroquois (Campeau 2003 : 313). À la fin du XVII siècle, les propositions de mise en commun des territoires sont d'ailleurs accompagnées de wampums et d'une chaudière (Parmenter 2010 : 228, 257).

Il est donc clair que l'établissement de relations fraternelles dans le cadre d'un traité de paix a pour corollaire la libre circulation des chasseurs sur l'ensemble des territoires occupés par les alliés. Celle-ci constitue une renonciation au droit des parties à contrôler l'accès à ces régions, contrairement à la situation qui prévalait auparavant. Cela ne signifie toutefois pas que la notion de territoire national soit entièrement abandonnée. En effet, après la paix, un autochtone peut chasser sur le territoire de ses anciens 
ennemis sans crainte d'être agressé; en revanche, il ne peut y conduire une expédition commerciale ou s'y établir de manière durable sans obtenir une autorisation. Les traités généraux de 1665 et de 1701 reposeront sur ces principes; la métaphore du plat commun continuera d'ailleurs d'être employée jusqu'au XIX ${ }^{\mathrm{e}}$ siècle (voir Morin 2003; Beaulieu et al. 2013 : 115-132 et 200-211) ${ }^{4}$. Par ailleurs, après l'établissement des réfugiés Wendats dans la région de Québec en 1651, ceux-ci semblent avoir négocié les limites de leurs territoires de chasse avec les Algonquins et, selon toute vraisemblance, avec les Innus (Beaulieu et al. 2013 : 60-62, 120-127). Ces ententes particulières entre nations sédentaires écartaient peut-être le principe de la mise en commun des ressources, qui avait principalement pour objectif de mettre fin aux attaques iroquoises. Quoi qu'il en soit, à l'intérieur des territoires nationaux, les chefs de bande ont continué d'exercer un contrôle sur les territoires de chasse de la vallée du Saint-Laurent, conformément à des règles qui paraissent anciennes.

\section{Notes}

1. Dans ce jugement, la Cour suprême conclut que les activités traditionnelles de pêche et de chasse n'ont jamais été interdites sous le Régime français; à notre avis, en refusant de se prononcer sur la question du titre aborigène, elle laisse planer un doute sur une éventuelle extinction des droits territoriaux des peuples autochtones à cette époque (Leclair et Morin 2011, paragr. 139-140)

2. En 1620 , il fait lire publiquement la commission du vice-roi et la sienne, puis tirer du canon. Il affirme avoir ainsi pris « possession de l'habitation et du pays au nom » du « vice-roi »; un procès-verbal est alors dressé (Champlain 2009: 17). Cette prise de possession semble résulter uniquement du changement de titulaire de l'autorité royale, sans affecter l'étendue du territoire revendiqué par la France.

3. Voir aussi un passage obscur où Radisson semble dire que des Outaouais affamés, qui se sont réfugiés sur un territoire voisin, ont été dépouillés de leurs provisions par ceux qui chassaient avec lui à cet endroit (Warkentin 2012 : 269).

4. Beaulieu et al. (2013) soulignent qu'au début du XIX ${ }^{e}$ siècle plusieurs nations autochtones de la vallée du Saint-Laurent refusent de reconnaître à leurs partenaires un droit d'accès à leurs territoires de chasse. Néanmoins, les Hurons et les Iroquois admettent que ceux-ci ont fait l'objet d'une mise en commun sous le Régime français. Leurs conceptions semblent donc avoir évolué par la suite.

\section{Législation et jurisprudence citées}

Behn c. ulton Contracting Ltd., [2013] CSC 26, paragr. 32-36.

Loi constitutionnelle de 1982, annexe B, ch. 11 .

R. c. Côté, [1996] 3 R.C.S. 139, paragr. 42-54.

R. c. Marshall; R. c. Bernard, [2005] 2 R.C.S. 220, 2005 CSC 43, paragr. 65-70.

Manitoba Metis Federation Inc. c. Canada (Procureur général), [2013] CSC 14, paragr. 41-45.

\section{Archives}

ANF (Archives nationales de France), 1706a: «Faits et articles [...] ». A.N.F. col., C11A, vol. 25, fo 44-44r.

—, 1706b: «Jacques Raudot [...]». A.N.F. col., Cl1A, vol. 25, fo $33-36 r$.

—, 1707 : «Copie de l'original de la requête du Sr. Hazeur [...] ». A.N.F. col., Cl1A, vol. 59, fo $297 \mathrm{v}-298 \mathrm{v}$.

\section{Ouvrages cités}

ANKER, Kirsten, 2014 : « Translating Sui Generis Aboriginal Rights in the Civilian Imagination », in R. Tremblay et L. Smith (dir.), Les intraduisibles en droit civil : 1-36. Éditions Thémis, Montréal.

ARNEIL, Barbara, 1996 : John Locke and America. Clarendon Press, Oxford.

Arrêts et Règlements du Conseil supérieur de Québec et Ordonnances et jugements des Intendants du Canada, 1855. E.R. Fréchette, Québec.

BAILEY, Alfred G., 1969: The Conflict of European and Eastern Algonkian Cultures 1504-1700: A Study of Canadian Civilization. University of Toronto Press, Toronto et Buffalo.

BARNARD, Alan, et James WOODBURN, 1988: «Property, Power and Ideology in Hunting-Gathering Societies: an Introduction », in T. Ingold, D. Riches et J. Woodburn, Hunters and Gatherers, vol. 2, Property, Power and Ideology: 4-31. Berg Publishers, Oxford et Washington.

BEAULIEU, Alain, 2008 : «"Lon a point d'ennemis plus grands que ces sauvages". L'Alliance franco-innue revisitée ». Revue d'histoire de l'Amérique française 61(3-4) : 365-395.

-, 2013: "An equitable right to be compensated": The Dispossession of the Aboriginal Peoples of Quebec and the Emergence of a New Legal Rationale (1760-1860)». The Canadian Historical Review 94(1) : 1-27.

BEAULIEU, Alain, S. BÉREAU et J. TANGUAY, 2013 : Les Wendats du Québec. GID, Québec.

BENDER, Barbara, et Brian MORRIS, 1988: « Twenty years of history, evolution and social change in gatherer-hunter studies », in T. Ingold, D. Riches et J. Woodburn, Hunters and Gatherers, vol. 1, History, Evolution and Social Change: 4-14. Berg Publishers, Oxford and Washington.

BIDEAUX, Michel, 1986: Jacques Cartier. Relations. Presses de l'Université de Montréal, Montréal.

BISSONNETTE, Alain, 2007 : «Un regard d'anthropologue sur le dialogue entre les traditions juridiques notamment en matière de rapports au territoire », in Myriam Jézéquel (dir.), La justice à l'épreuve de la diversité culturelle: 191-232. Yvon Blais, Cowansville.

BOUCHARD, Russel, 2002: L'exploration du Saguenay par J.L. Normandin en 1732: Au cœur du Domaine du Roi. Septentrion, Sillery.

BOUCHER, Pierre, 1964 : Histoire véritable et naturelle des mours et productions du pays de la Nouvelle-France vulgairement dite le Canada, 1664. Société historique de Boucherville, Boucherville.

BRANDAO, Jose Antonio, 1997 : "Your Fyre shall Burne no more", Iroquois Policy toward New France and its Native Allies to 1701. University of Nebraska Press, Lincoln

BRUN, Henri, 1974 : Le territoire du Québec : six études juridiques. Presses de l'Université Laval, Québec. 
CAMPEAU, Lucien (dir.), 1967 : Monumenta Novae Franciae, vol. I, La première mission d'Acadie (1602-1616). Apud « Monumenta Hist. Soc. Iesu »/Presses de l'Université Laval, Rome/Québec.

—, 1979: Monumenta Novae Franciae, vol. II, Établissement à Québec (1616-1634). Apud « Monumenta Hist. Soc. Iesu»/ Presses de l'Université Laval, Rome/Québec.

—, 1987 : Monumenta Novae Franciae, vol. III, Fondation de la mission huronne (1635-1637). Apud « Monumenta Hist. Soc Iesu »/Presses de l'Université Laval, Rome/Québec.

—, 1989 : Monumenta Novae Franciae, vol. IV, Les grandes épreuves (1638-1640). Institutum Societatis Iesu/Bellarmin, Rome/Montréal.

—, 1990a: Monumenta Novae Franciae, vol. V, La bonne nouvelle reçue (1641-1643). Institutum Historicum Societatis Iesu/ Bellarmin, Rome/Saint-Laurent.

—, 1990b : Monumenta Novae Franciae, vol. VI, Recherche de la paix (1644-1646). Institutum Historicum Societatis Iesu/ Bellarmin, Rome/Saint-Laurent.

—, 1994 : Monumenta Novae Franciae, vol. VII, Le témoignage du sang (1647-1650). Institutum Historicum Societatis Iesu/ Bellarmin, Rome/Saint-Laurent.

—, 1996 : Monumenta Novae Franciae, vol. VIII, Au bord de la ruine (1651-1656). Institutum Historicum Societatis Iesu/Bellarmin, Rome/Saint-Laurent.

—, 2003: Monumenta Novae Franciae, vol. IX, Pour le salut des Hurons (1657-1661). Institutum Historicum Societatis Iesu/ Bellarmin, Rome/Saint-Laurent.

CARLOS, Ann M., et Frank D. LEWIS, 2006 : « Native American Property Rights in the Hudson Bay Region: A Case Study of the Eighteenth Century Cree », in T.L. Anderson, B.L. Benson et T.E. Flanagan (dir.), Self-Determination, The Other Paths for Native Americans : 4-93. Stanford University Press, Stanford.

CAVANAGH, Edward, 2014 : « Possession and Dispossession in Corporate New France, 1600-1663: Debunking a "Juridical History" and Revisiting Terra Nullius ». Law and History Review 32(1): 97-125.

CHAMBERLAND, Robert, J. LEROUX, S. AUDET, S. BOUILLÉ et M. LOPEZ, 2004 : Terra incognita des Kotakoutouemis. L'Algonquinie orientale au XVII siècle. Presses de l'Université Laval, Québec.

CHAMPLAIN, Samuel de, 2009: Premiers récits de voyages en Nouvelle-France 1603-1619. Réédition intégrale en français moderne, introduction et notes par Mathieu d'Avignon. Presses de l'Université Laval, Québec.

—, 2010 : Derniers récits de voyages en Nouvelle-France et autres récits 1620-1632. Réédition intégrale en français moderne, introduction et notes par Mathieu d'Avignon. Presses de l'Université Laval, Québec.

COLE, Daniel H., et Elinor OSTROM, 2012 : « The Variety of Property Systems and Rights in Natural Ressources », in D.H. Cole et E. Ostrom, Property in Land and Other Ressources : 37-66. Lincoln Institute of Land Policy, Cambridge.

COOK, Peter, 2007 : « Kings, Captains and Kin: French Views of the Native American Political Cultures in the Sixteenth and Early Seventeenth Century», in P.C. Mancall, The Atlantic World and Virginia: 307-341. University of North Carolina Press, Chapel Hill.

-, 2008: "Vivre comme frères": Native-French Alliances in the St Lawrence Valley, 1535-1667. Thèse de doctorat, département d'histoire, Université McGill, Montréal.

D'AVIGNON, Mathieu, 2008 : Champlain et les fondateurs oubliés. Presses de l'Université Laval, Québec.
DAWSON, Nelson-Martin, 2003 : Des Attikamègues aux Têtes-deBoule, Mutation ethnique dans le Haut Mauricien sous le Régime français. Septentrion, Sillery.

—, 2005 : Feu, fourrures, fléaux et foi foudroyèrent les Montagnais. Septentrion, Sillery.

—, 2011 : Fourrures et forêts métissèrent les Montagnais. Septentrion, Sillery.

DELÂGE, Denys, 1996 : «Les premières nations d'Amérique du Nord sont-elles à l'origine des valeurs écologiques et démocratiques contemporaines? » in L. Turgeon, D. Delâge et R. Ouellet (dir.), Transferts culturels et métissages Amériquel Europe, $X \mathrm{XI}^{e}-\mathrm{XX}$ siècle : 317-345. PUL, Québec.

DELÂGE, Denys, et Étienne GILBERT, 2004 : «Les Amérindiens face à la justice coloniale française dans le gouvernement de Québec, 1663-1759. II. Eau de vie, traite des fourrures, endettement, affaires civiles ». Recherches amérindiennes au Québec 34(1): 31-41.

DEMSETZ, Harold, 1967 : « Toward a Theory of Property Rights ». American Economic Review 57(2) : 347-359.

-, 2002 : «Toward a Theory of Property Rights II: The Competition Between Private and Collective Ownership ». The Journal of Legal Studies 31(S2) : S653-672.

—, 2011 : « Transaction Cost and the Organization of Ownership - An introduction ». Arizona Law Review 53(1) : 1-7.

DI ROBILANT, Anna, 2012 : «Common Ownership and Equality of Autonomy ». Revue de droit de McGill 58(2) : 263-320.

FEIT, Harvey A., 2004 «Les territoires de chasse avant leur "découverte"? Études et histoires sur la tenure, les incendies de forêt et la sociabilité de la chasse ». Recherches amérindiennes au Québec 34(3) : 5-21.

—, 2007: «Myths of the Ecological Whitemen: Histories, Science and Rights in North American-Native Relations », in M.R. Harkin et D.R. Lewis (dir.), Native Americans and the Environment, Perspectives on the Ecological Indian : 52-92. University of Nebraska Press, Lincoln et Londres.

—, 2009: «Histories of the Past, Histories of the Future: The Committed Anthropologies of Richard Slobodin, Frank G. Speck and Eleanor Leacock », in Richard Preston (dir.), A Kindly Scrutiny of Human Nature: Essays in Honour of Richard Slobodin : 45-76. Wilfrid Laurier University Press, Waterloo.

FLANAGAN, Tom, C. ALCANTARA et A. LE DRESSAY, 2010 : Beyond the Indian Act, Restoring Aboriginal Property Rights. McGill-Queen's University Press, Montréal et Kingston.

GAGNON, François-Marc (dir.), 2011 : The Codex Canadensis and the Writings of Louis Nicolas. Gilcrease Museum/McGill-Queen's University Press, Tulsa/Montréal et Kingston.

GANONG, W.F., 1908 : The Description and Natural History of the Coasts of North America (Acadia) by Nicolas Denys. Champlain Society, Toronto.

GÉLINAS, Claude, 2000 : La gestion de l'étranger. Les Attikamekw et la présence eurocanadienne en Haute-Mauricie, 1760-1870. Septentrion, Sillery.

GRAMMOND, Sébastien, 2003: Aménager la coexistence, Les Peuples autochtones et le droit canadien. Bruylant/ Yvon Blais, Bruxelles/Cowansville.

GREER, Allan, 2012: «Commons and Enclosure in the Colonization of North America ». American Historical Review $117(2)$ : 365-386. 
GREER, Allan, et C. DESBARATS, 2011 : « Où est la NouvelleFrance? » Revue d'histoire de l'Amérique française 64(3-4) : 31-62.

HARDIN, Garret, 1968: "The Tragedy of the Commons». Science, N.S. 162(3859): 1243-1248.

HAVARD, Gilles, 2003 : Empire et Métissage, Indiens et Français dans le Pays d'en Haut, 1660-1715. Septentrion/Presses de l'Université Paris Sorbonne. Sillery/Paris.

HENNEPIN, Louis, 1683a: Description de la Louisiane, nouvellement decouverte au Sud'Oüest de la Nouvelle France, par ordre du roy. Avec la carte du pays: Les moeurs \& la maniere de vivre des sauvages. Chez la veuve Sebastien Huré, Paris.

—, 1683b: Les mours \& la maniere de vivre des sauvages, dans Description de la Louisiane, nouvellement decouverte au Sud'Oüest de la Nouvelle France [...]. Chez la veuve Sebastien Huré, Paris ( ${ }^{\mathrm{e}}$ ouvrage avec pagination indépendante).

HOUDE, Nicolas, 2011 : "Experimenting with what will become our traditions": Adaptive co-management as a bridge to an Atikamekw Nehirowisiw post-treaty world in Nitaskinan, Canada. Thèse de doctorat, département de géographie, Université McGill, Montréal.

HUPPÉ, Luc, 2009 : «L'établissement de la souveraineté européenne au Canada ». Les Cahiers de droit 50(11) : 153-206.

JR (voir THWAITES 1896-1901).

KRECH III, Shepard, 1999 : The Ecological Indian Myth and History. W.W. Norton, New York et Londres.

KRIER, James E., 2009 : "Essay - Evolutionary Theory and the Origin of Property Rights ». Cornell Law Review 95(1) : 139-159.

LACASSE, Jean-Paul, 2004: Les Innnus et le territoire, Innu Tipenitamun. Septentrion, Sillery.

LACASSE, Jean-Paul, et Valérie CABANES, 2009 : « Les fondements de l'ordre juridique Innu : la parole des aînés comme source de connaissance », in M. D'Avignon et C. Girard (dir.), A-t-on oublié que jadis nous étions "frères »: 143-162. PUL, Québec.

LAFITAU, Joseph François, 1724 : Mœurs des sauvages ameriquains comparées aux moeurs des premiers temps. 4 tomes. Saugrain l'aîné/Charles-Estienne Hochereau, Paris.

LAHONTAN, Baron de, 1990a: Euvres complètes I. Édition critique par Réal Ouellet et Alain Beaulieu. PUM, Montréal.

—, 1990b : Euvres complètes II. Édition critique par Réal Ouellet et Alain Beaulieu. PUM, Montréal.

LAJOIE, Andrée, J.-M. BRISSON, S. NORMAND et A. BISSONNETTE, 1996 : Le statut juridique des peuples autochtones au Québec et le pluralisme juridique. Yvon Blais, Cowansville.

LA POTHERIE, Leroy Bacqueville de, 1997a [1721] : Histoire de l'Amérique Septentrionale. Tome 1. Éditions du Rocher, Paris.

—, 1997b [1721] : Histoire de l'Amérique Septentrionale. Tome 2, Éditions du Rocher, Paris.

LAVOIE, Michel, 2010a : Le Domaine du Roi 1652-1859. Septentrion, Québec.

—, 2010b : C'est ma seigneurie que je réclame, La lutte des Hurons de Lorette pour la seigneurie de Sillery, 1650-1900. Boréal, Montréal.

LEACOCK, Eleanor B., 1974 : The Montagnais "Hunting Territory" and the Fur Trade. Kraus Reprint, Milwood.

—, 1980 : «Les relations de production parmi les peuples chasseurs et trappeurs des régions subarctiques du Canada ». Recherches amérindiennes au Québec X(1-2): 79-89.

LECLAIR, Jean, et Michel MORIN, 2011 : «Peuples autochtones et droit constitutionnel ». JurisClasseur Québec. coll. « Droit public», Droit constitutionnel (fasc. 15). LexisNexis Canada, Montréal.
LECLERQ, Chrestien, 1691 : Premier etablissement de la foy dans la Nouvelle-France. 2 tomes. Amable Auroy. Paris.

—, 1999 : Nouvelle relation de la Gaspésie. PUM, Montréal.

LEROUX, Jacques, 2009 : « Éthique et symbolique de la responsabilité territoriale chez les peuples algonquiens du Québec ». Recherches amérindiennes au Québec 29(1-2) : 85-97.

—, 2010 : «Le contrôle territorial ou posséder ce que l'on protège chez trois peuples algonquiens du Québec », in Pierre Noreau (dir.), Gouvernance autochtone : reconfiguration d'un avenir collectif : 99-131. Thémis, Montréal.

LESCARBOT, Marc, 1610 : La conversion des sauvages qui ont esté baptizés en la Nouvelle France, cette année 1610: avec un bref récit du voyage du sieur de Poutrincourt. Jean Millot, Paris.

—, 1907 : History of New France. Champlain Society, Toronto.

—, 2007 : Voyages en Acadie (1604-1607) suivis de La description des mours souriquoises comparées à celles d'autres peuples. Édition critique par Marie-Christine Pioffet. PUL, Québec.

LESTRINGANT, Frank, 2004: Le Huguenot et le sauvage. Droz, Genève.

LOCKE, John, 2002: The Second Treatise on Government and A Letter Concerning Toleration. Dover Publications, Mineola.

MAILHOT, José, 1985: «La mobilité territoriale chez les Montagnais-Naskapis du Labrador $»$. Recherches amérindiennes au Québec 15(3) : 3-11.

_, 1993: Au pays des Innus. Les gens de Sheshatshit. Recherches amérindiennes au Québec, Montréal.

MAILHOT, José, et Sylvie VINCENT, 1982: « Le droit foncier montagnais ». Interculture 15(2-3): 65-74.

MORANTZ, Toby, 1986: «Historical Perspective on Family Hunting Territories in Eastern James Bay ». Anthropologica n.s. 28(1-2) : 64-91.

MORIN, Michel, 1995 : « Un document inédit sur la portée territoriale de la Proclamation royale : les notes des juges de la Cour suprême dans l'affaire R. c. Bonhomme ». Revue générale de droit $26: 557-564$

—, 1997 : L'Usurpation de la souveraineté autochtone. Boréal, Montréal.

—, 2003 : « Manger avec la même micoine dans la même gamelle : à propos des traités conclus au Québec avec les Amérindiens (1665-1760)». Revue générale de droit 33 : 93-129.

—, 2010 : «Des nations libres sans territoire? Les Autochtones et la colonisation de l'Amérique française $\mathrm{du} \mathrm{XVI}^{\mathrm{e}}$ au XVIII ${ }^{\mathrm{e}}$ siècle ». Revue d'histoire du droit international public 12(1) : 1-70.

—, 2013 : «Fraternité, souveraineté et autonomie des Autochtones en Nouvelle-France ». Revue générale de droit 43(2) : 531-598.

PARMENTER, Jon, 2010 : The Edge of the Woods. Michigan State University Press, East Lansing.

PERROT, Nicolas, 2007 : Mémoire sur les mours, coustumes et relligion des sauvages de l'Amérique septentrionale. LUX éditeur, Montréal.

PROMISLOW, Janna, 2008 : « One Chief, Two Chiefs, Red Chiefs, Blue Chiefs Newcomer Perspectives on Indigenous Leadership in Rupert's Lands and the North-West Territories », in H. Foster, B.L. Berger et A.R. Buck (dir.), The Grand Experiment Law \& Legal Culture in British Settler Societies: 55-77. UBC Press, Vancouver.

—, 2010 : " "Thou Wilt Not Die of Hunger ... for I Bring Thee Merchandise". Consent, Intersocietal Normativity, and the Exchange of Food at York Factory, 1682-1763 », in J. Webber et C.M. MacLeod (dir.), Between Consenting Peoples, Political 
Community and the Meaning of Consent: 77-114. UBC Press, Vancouver.

RADISSON, Pierre-Esprit, 1999 : Les aventures extraordinaires d'un coureur des bois. Nota bene, Québec.

RAUDOT, Antoine-Denis, 1904 : « Lettre XXXVIII ${ }^{e}$ de la chasse d'hiver des sauvages et de leurs sueries ", in Camille de Rochemonteix (dir.), Relations par lettres de l'Amérique Septentrionalle, (années 1709 et 1710) : 96-98. Letouzey et Ané, Paris. <http://gallica. bnf.fr/ark:/12148/bpt6k57745455/f165.image> (consulté le $1^{\text {er }}$ avril 2014).

SACY, Samuel de (dir.), 1962 : Les Essais de Michel de Montaigne. Club français du livre, Paris.

SAGARD, Gabriel Theodat, 1866 [1636] : Histoire du Canada. 4 vol., Tross, Paris.

SAVARD, Rémi, 1996: L'Algonquin Tessouat et la fondation de Montréal. L'Hexagone, Montréal.

—, 2004 : La forêt vive. Boréal, Montréal.

SLATTERY, Brian, 1979: The Land Rights of Indigenous Canadian Peoples as Affected by the Acquisition of Their Territories. University of Saskatchewan Native Law Center, Saskatoon.

SPECK, Frank G., 1926 : « Land ownership among hunting peoples in primitive America and the world's marginal areas ». Atti des XXII Congresso internazionale degli Americanisti, Roma, settembre 1926 : 323-332. Kraus Reprint, Nendeln.

SPECK, Frank G., et Loren C. EISELEY, 1942 : « MontangaisNaskapi Bands and Family Hunting Districts in the
Southeastern Labrador Peninsula ». Proceedings of the American Philosophical Society 85(2) : 215-242.

THWAITES, Reuben Gold (dir.), 1896-1901 : The Jesuits and Allied Documents. 71 vol. Burrows Brothers, Cleveland.

TOMLINS, Christopher, 2010: Freedom Bound. Cambridge University Press, Cambridge.

VIAU, Roland, 1997 : Enfants du néant et mangeur d'âmes, Guerre, culture et société en Iroquoisie ancienne. Boréal Compact, Montréal.

VINCENT, Sylvie, 2010 : «Circulation sur le territoire et régime foncier des Innus dans la première moitié du $\mathrm{xx}^{\mathrm{e}}$ siècle », in Pierre Noreau (dir.), Gouvernance autochtone: reconfiguration d'un avenir collectif : 135-154. Thémis, Montréal.

VIVIER, Nadine, 2003: «Les biens communaux en France », in Marie-Danielle Demélas et N. Vivier (dir.), Les propriétés collectives face aux attaques libérales (1750-1914). Presses universitaires de Rennes, Rennes.

WARKENTIN, Germaine (dir.), 2012 : Pierre-Esprit Radisson, The Collected Writings, vol. I, The Voyages. McGill-Queen's University Press/Champlain Society, Montréal-Kingston/Londres/Ithaca.

WARWICK, Jack, 1997 : Gabriel Sagard. Le grand voyage du pays des Hurons suivi du Dictionaire de la langue huronne. PUM, Montréal.

YIRUSH, Craig, 2011 : Settlers, Liberty and Empire. Cambridge University Press, Cambridge.

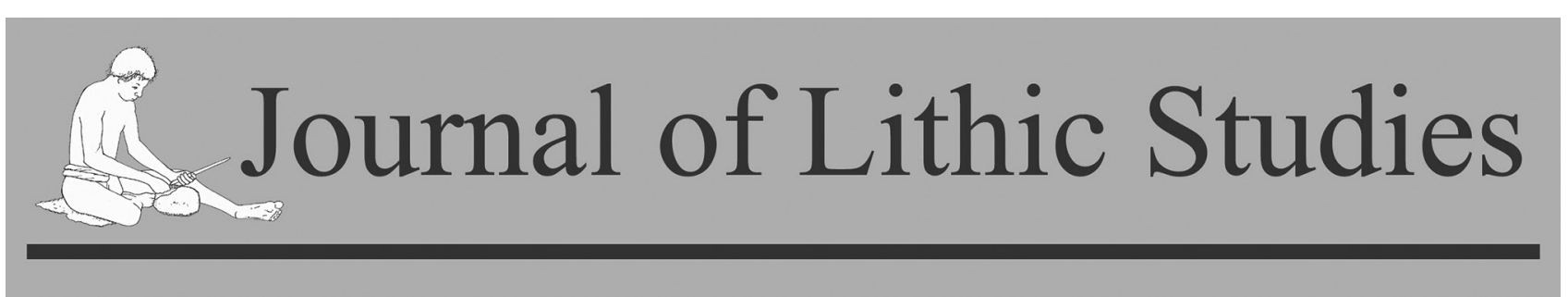

Nous sommes heureux de vous annoncer la parution du premier numéro du Journal of Lithic Studies. Ce premier numéro se concentre sur la publication de communications présentées lors de l'International Symposium on Chert and Other Knappable Materials en 2013. Les articles couvrent un large éventail de sujets tels que l'analyse des traces d'usure, la caractérisation des matières premières et la recherche dans la structure des échanges. Ce numéro présente également différentes recherches menées en Amérique du Nord, en Europe, au Proche-Orient et en Asie de l'Est. La version intégrale est disponible sur Internet : http://journals.ed.ac.uk/lithicstudies/issue/view/66.

Journal of Lithic Studies accepte présentement des articles pour le second numéro - qui devrait être publié en septembre 2014 et réunir des articles sur les recherches archéologiques portant sur la fabrication et l'utilisation des outils en pierre ainsi que sur l'origine et les propriétés des matières premières utilisées dans leur production. Les recherches de toutes les régions géographiques et de toutes les périodes chronologiques seront considérées. Même si les numéros sont publiés en mars et septembre, les articles soumis peuvent être rapidement mis en ligne une fois qu'ils auront été acceptés. Journal of Lithic Studies est publié sur Internet et son contenu est disponible gratuitement. Pour plus d'informations : http://journals.ed.ac.uk/lithicstudies. 